\title{
Positron Annihilation Spectroscopy to Characterize Irradiation Induced Vacancy Type Defects in Materials for Nuclear Fission and Fusion
}

\author{
Marie-France BARTHE ${ }^{1}$ \\ ${ }^{1}$ CEMHTI, CNRS, Université d'Orléans (Orléans, FRANCE)
}

In nuclear reactors, materials are submitted to irradiations and modification of their macroscopic properties such as swelling or hardening is observed... It is of first importance to understand the origin of these evolutions and how damage at the atomic scale such as vacancy and interstitials type defects can interact with each other or with solutes and impurities to make the microstructure evolved. So the properties of defects have to be determined.

Positron annihilation spectroscopy (PAS) is a well-established technique to characterize materials [1]. Due to its positive charge, positron is sensitive to the local variations of the Coulomb potential in solids. As the anti-particle of the electron, both particles can annihilate leading to the emission of gamma rays with energy depending on the momentum of the electron positron annihilated pair. The detection of these gamma rays arises original information on the defects because positron can be trapped and annihilate in localized state such as vacancies. Two different and complementary annihilation characteristics can be measured. Firstly Positron Annihilation Lifetime Spectrometer (PALS) allows to measure the positron lifetime which depends on the local electron density at the annihilation site. The second characteristic is the momentum distribution of the electron positron annihilated pairs and is obtained by measuring the energy spectrum of the gamma annihilation rays using a Doppler Broadening Spectrometer (DBS). Both characteristics give the signature of the defects and allow determining some of their properties such as their nature, concentration, chemical environment... By using slow positron accelerators which produce monokinetic positrons beams with energy varying from 0.1 to $30 \mathrm{keV}$ it is possible to study the depth profile of defects in thin layers from 0.1 to about $5 \mu \mathrm{m}$ depending on the density of materials with a resolution of the order of $0.1 \mathrm{xdepth}$. ${ }^{22} \mathrm{Na}$ source based beams [2] or user facilities taking advantages of high positrons flux available around nuclear reactor (FRMII, Garching [3]) or LINAC( as in AIST in Japan [4]) are now available to study defects in materials. PAS has numerous advantages among them, it is non-destructive and can be used for conducting as well as insulating materials crystalline or amorphous. This technique is especially useful to probe vacancy defects in metals for which it is one of the only direct characterization. The annihilation characteristics can be predicted by first principle calculations and these data when available can help to identify defects. Sensitive to the single vacancy, PAS can allow to determine the size of the vacancy clusters up to a maximum of $1 \mathrm{~nm}$ approximately in the concentration range from $10^{15}$ to a few $10^{18} \mathrm{~cm}^{-3}$.

In nuclear science this technique is very powerful to characterize not only damage induced by irradiation but also to determine some fundamental properties of defects that are required for modeling of the microstructural evolution of materials such as formation, migration, agglomeration, interaction with solutes, impurities or transmutation products such as Helium or Hydrogen. PAS has already demonstrated its major role in the study of vacancy defects and material related properties. For example the results obtained by Vehanen et al [6] have had an outmost impact on the knowledge of the role of carbon on vacancy behavior in Fe. They have demonstrated the trapping of vacancy in Carbon Vacancy complexes and the non-localization of $C$ atoms in the center of the vacancy. This attractive interaction and localization has been confirmed by ab initio calculations [7]. It has also to be underlined that positrons can be trapped in precipitates if its affinity for the material of precipitate is higher than the one of the matrix. Nagai et al have demonstrated the complementarity of positron techniques with Atomic Probe Tomography and have recently underlined the effects of matrix damage probed by PAS on the hardening [5]. 
In this work we will report different results on the determination of the properties of vacancy defects in $\mathrm{W}$ and $\mathrm{UO}_{2}$ using separate effects irradiation experiments coupled to PAS experimental measurements and theoretical data. Recently the self-consistent two components Density Functional Theory has been implemented in PAW approximation and has allowed circumcising the nature of the detected vacancy defects in $\mathrm{UO}_{2}$ irradiated with electrons or alpha particles [8]. The comparison of the experimental positron lifetime with calculated values show that the Schottky defects made of one uranium vacancy associated to 2 oxygen vacancies seem to be the most detected defects. Due to its properties tungsten has been chosen to cover part of the divertor in ITER and is envisaged for first walls in next tokamak generation such as DEMO. In order to better understand its swelling and embrittlement observed in special irradiation conditions, we use PAS to study the vacancy defects properties in this material. We have first shown the migration and agglomeration of single vacancy in the temperature range from 473 to $600 \mathrm{~K}$ [9] and its interaction with helium [10]. More recently we have observed the formation of vacancy clusters in self ion irradiated $W$ and the effect of the temperature on the size of these clusters. Today, the development of positron microbeams is very promising. It has already allowed to show the creation of vacancies in region close to the fracture zone of $\mathrm{H}$ charging stainless steel [11].

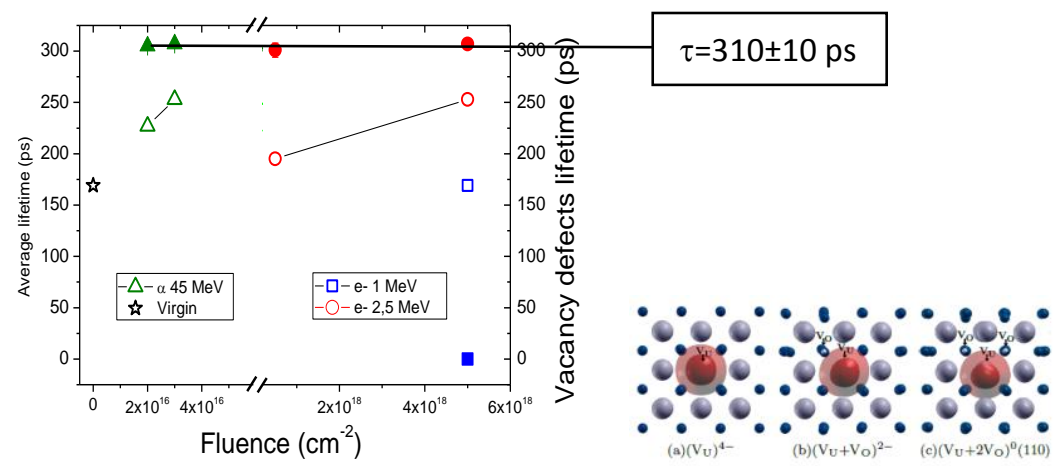

Fig. 1 : Positron Lifetime in electron and alpha irradiated $\mathrm{UO}_{2}$ and positron isodensities in Uranium vacancy and complexes with oxygen vacancy as calculated in self-consistent 2 components Density Fonctionnal Theory [8]

\section{References}

[1] M.-F. Barthe et al., Techniques de l'Ingénieur P3(P145) 2610 (2003).

[2] P Desgardin et al.," Slow positron beam facility in Orleans", Materials science Forum 363, 523 (2001).

[3] https://www.frm2.tum.de/en/the-neutron-source/secondary-sources-for-neutrons-and-positrons/ positron-source/

[4] https://unit.aist.go.jp/rima/xr-pos/group detail/facilities/facilities.html

[5] A. Kuramoto et al., "Microstructural changes in a Russian-type reactor weld material after neutron irradiation, post-irradiation annealing and re-irradiation studied by atom probe tomography and positron annihilation spectroscopy", Acta Materialia 61, 5236 (2013).

[6] A. Vehanen et al., "Vacancies and carbon impurities in a-Fe- electron irradiation", Phys. Rev. B 25, 762, (1982).

[7] C. Barouh et al.," Interaction between vacancies and interstitial solutes (C, N, and $O)$ in a -Fe: From electronic structure to thermodynamics", Phys. Rev. B 90, 054112 (2014).

[8] J. Wiktor et al., "Coupled experimental and DFT plus $\mathrm{U}$ investigation of positron lifetimes in $\mathrm{UO}_{2}$ ", Phys. Rev. B 90, 184101 (2014).

[9] P. E. Lhuillier et al., "Positron annihilation studies on the nature and thermal behaviour of irradiation induced defects in tungsten", Physica Status Solidi C 6, 2329 (2009).

[10] P. E. Lhuillier et al., "Helium retention and early stages of helium-vacancy complexes formation in low energy helium-implanted tungsten", JNM 433, 305 (2013).

[11] N. Oshima et al., Rapid three-dimensional imaging of defect distributions using a high-intensity positron microbeam", Appl. Phys. Lett. 94, 194104 (2009). 
Positron Annihilation Spectroscopy to Characterize Irradiation Induced Vacancy Type Defects in Materials for Nuclear Fission and Fusion

\author{
M-F. Barthe
}

CEMHTI, CNRS/Orléans University, 3A Rue de la Férollerie, 45071 Orléans, France

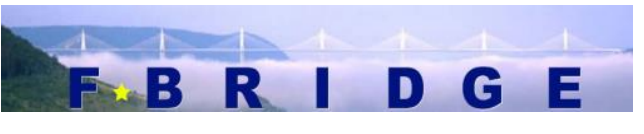




\section{CEMHTI Facilities for the study of irradiation in Materials}

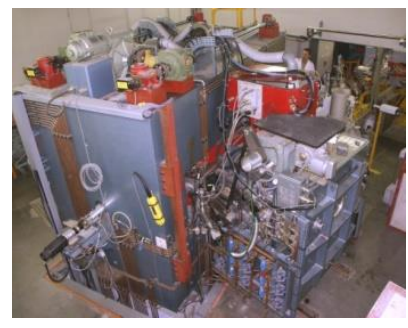

Cyclotron
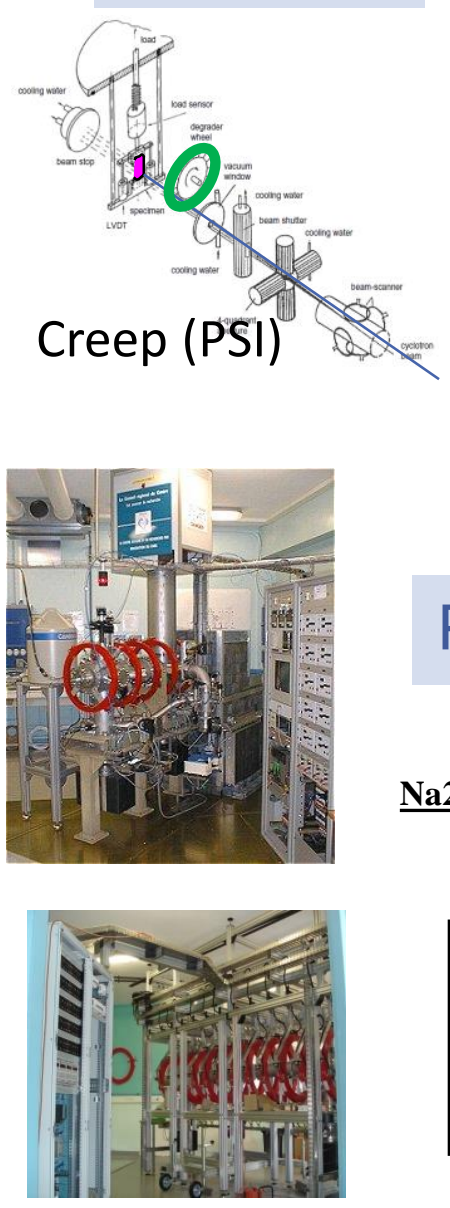

\section{emis.}

Light ions : $\mathrm{H}^{+}, \mathrm{D}^{+}, \alpha$

- Energy $=10-45 \mathrm{MeV}$

- Temperatures $-120^{\circ}$ to $1200^{\circ} \mathrm{C}$ DIAMANT

- Neutrons flux $\Rightarrow$ a few $10^{11}$ neutron $/ \mathrm{cm}^{2} / \mathrm{s}$
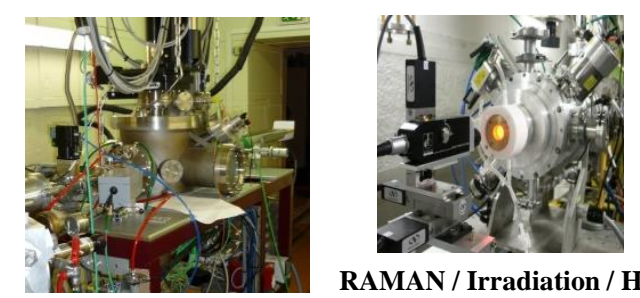

DIAMANT

Radiolysis, corrosion
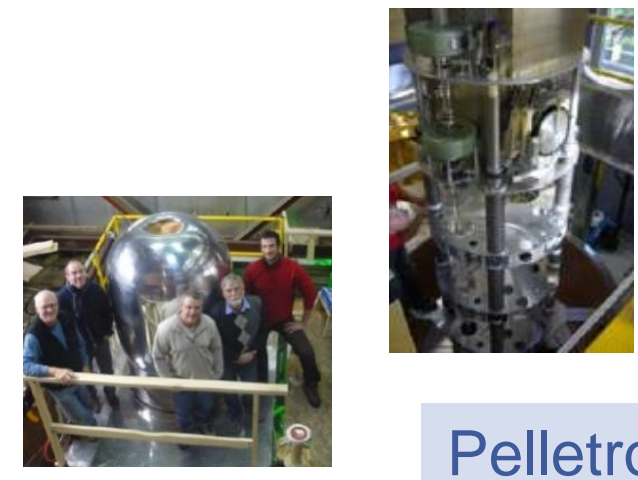

Particles beams :

Irradiations, and chemical and structural characterization

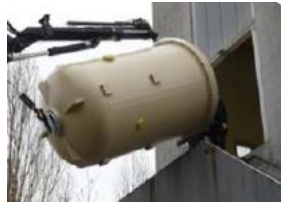

Feb. - Oct. 2014 :

Now running emis.

\section{Pelletron New}

Na22. Slow positron beam $(0.5-25 \mathrm{keV})$

- vacancy defects

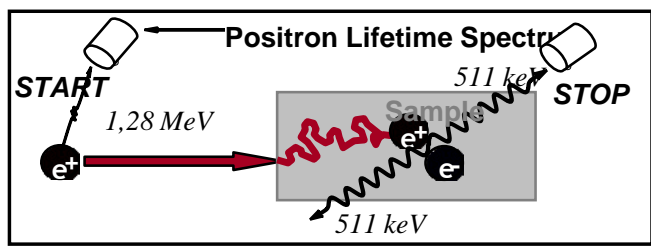

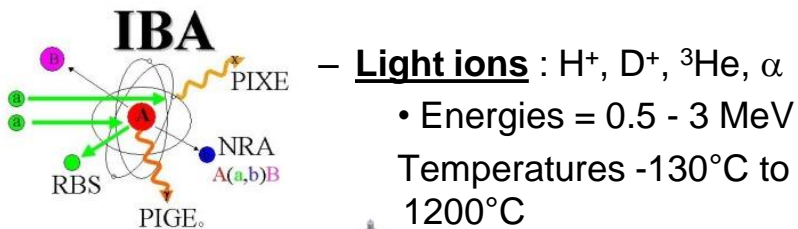

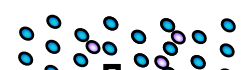

$\therefore: 58:$ $\therefore$ $\therefore: \div \circ:$ PIGE。
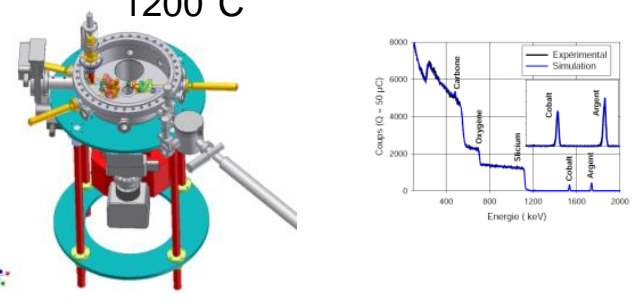

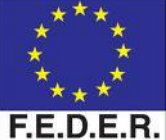




\section{Positron Annihilation Spectroscopy}

Positron $\mathrm{e}^{+}=$antiparticule of electron (opposite charge, same mass)

Positron Annihilation Spectroscopy is based on 2 properties of positron

$\checkmark$ In dense solids annihilation leads to emission of $2 \gamma: E=511 \mathrm{keV} \pm \Delta \mathrm{E}$

$\checkmark$ Trappping in vacancy defects

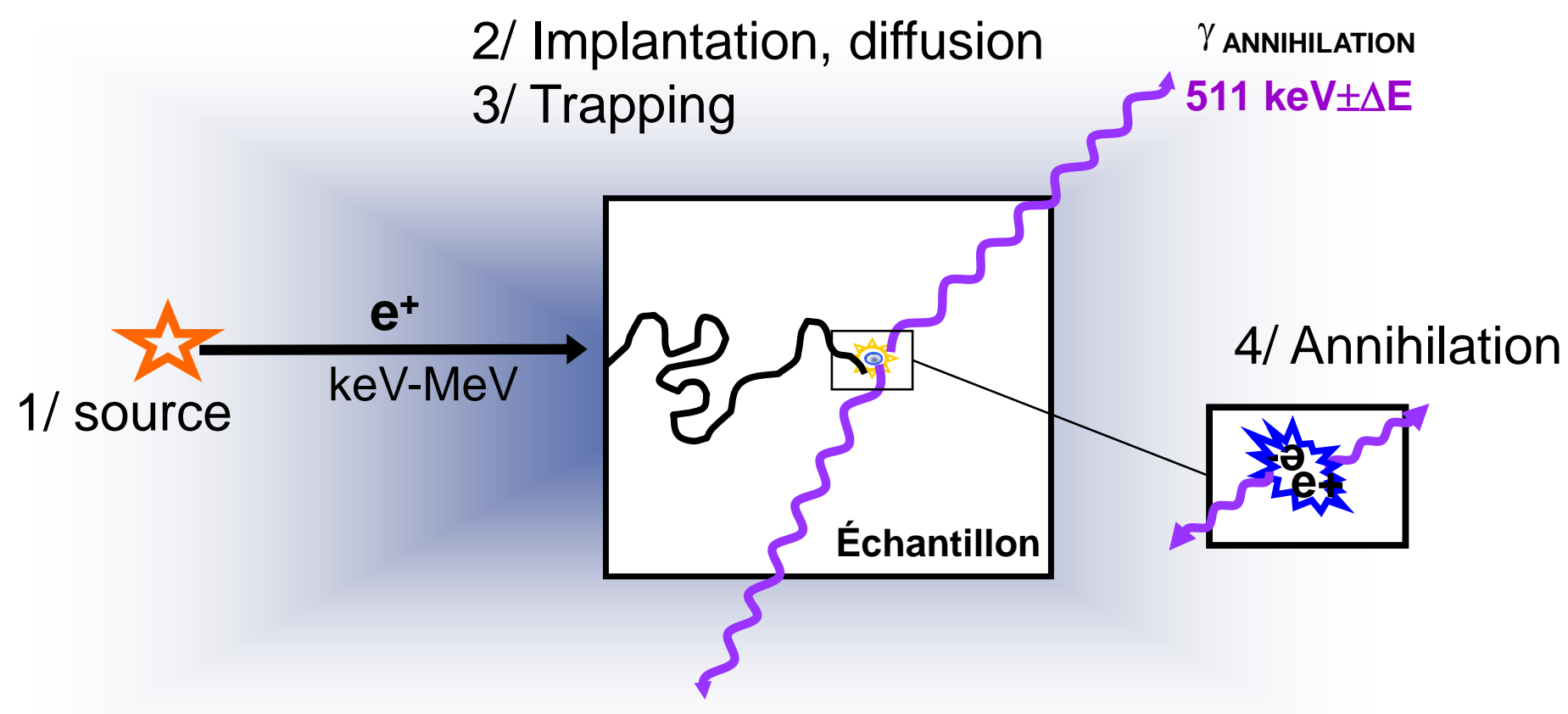

Electronic Structure of solids

$\longrightarrow$ Probe for vacancy defects (negative or neutral) and free volumes 


\section{Positron Source (1) : ${ }^{22} \mathrm{Na} \quad$ At CEMHTI}

${ }^{22} \mathrm{Na}$ : fast positrons

$\mathrm{T}_{1 / 2}=2.6$ ans,
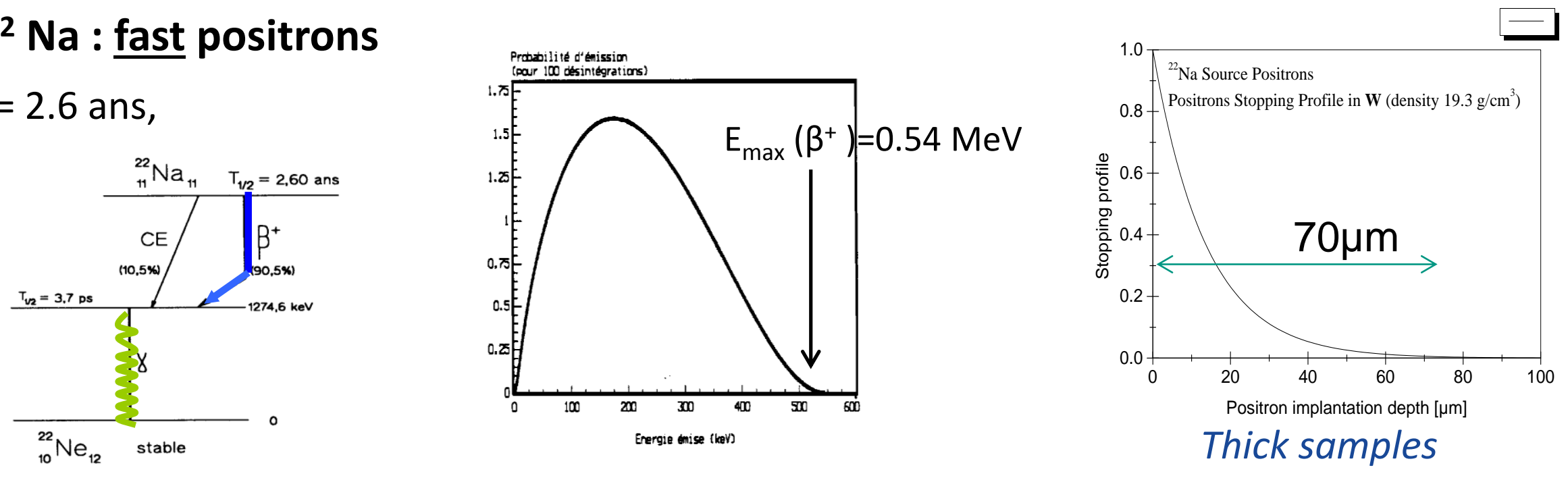

Slow positron beam: monokinetic slow positrons $(0.5-25 \mathrm{keV})$
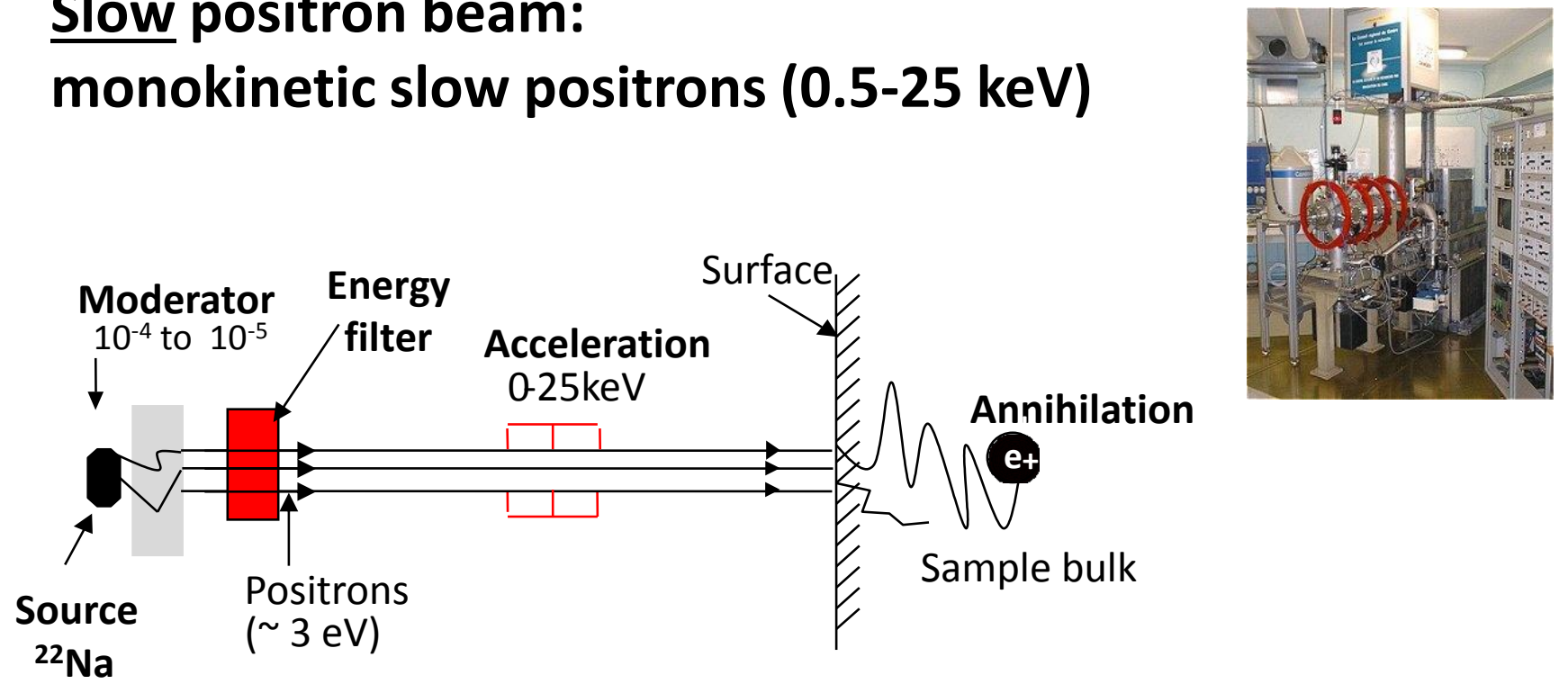

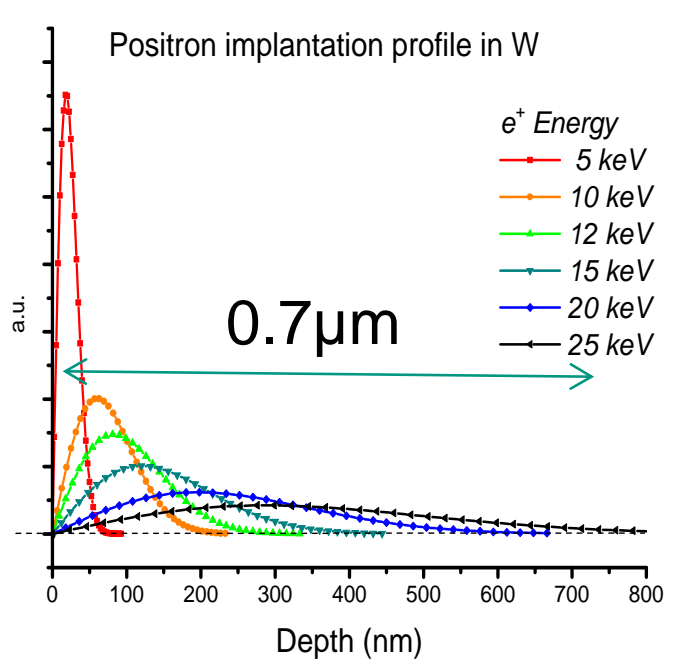

Thin layers 


\section{Doppler broadening spectrometry}

$$
\begin{gathered}
\Delta E=c P_{L} / 2 \\
P_{L}: \text { e- e+ pair } \\
\text { momentum }
\end{gathered}
$$

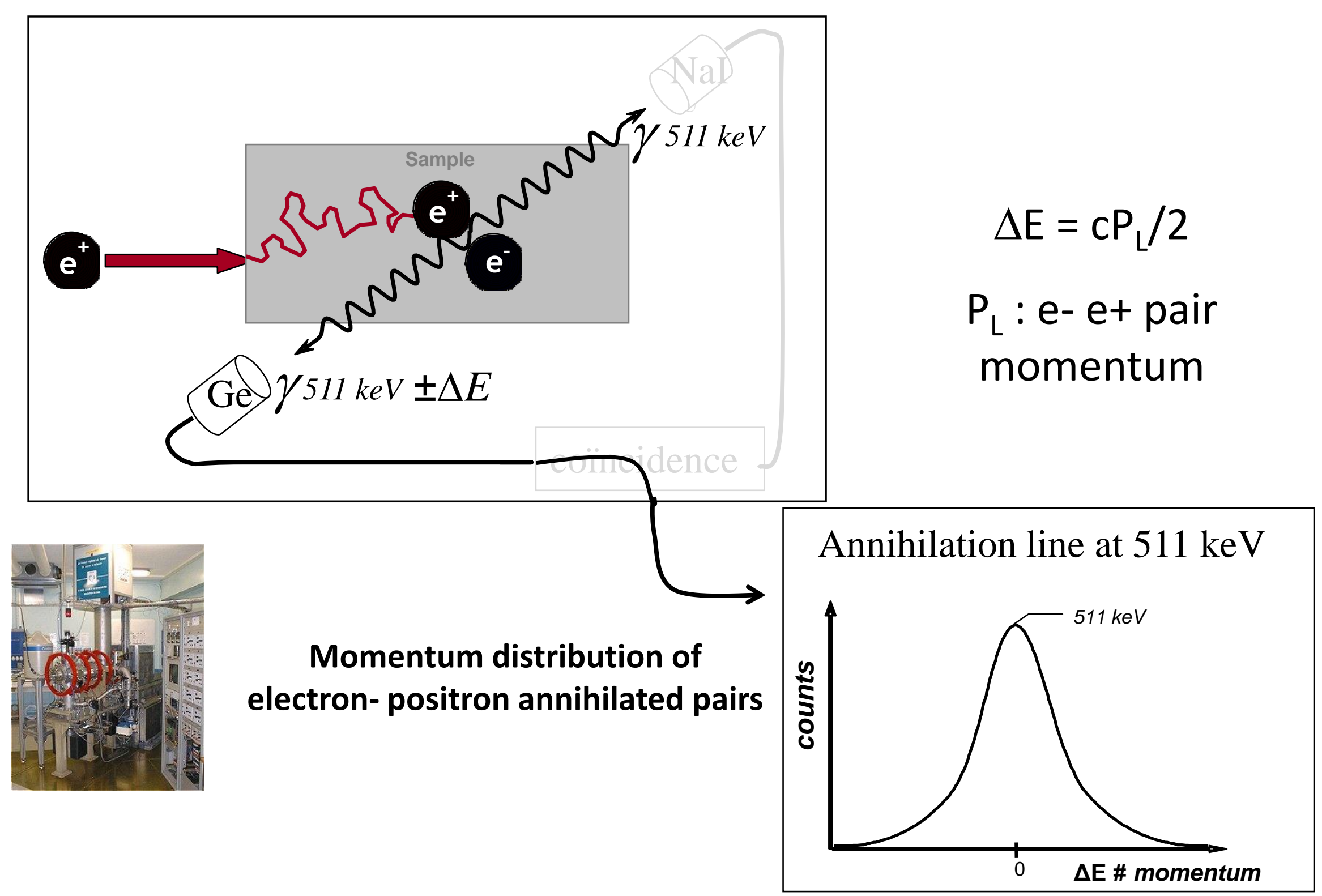




\title{
Doppler broadening spectrometry
}

\author{
$D E=C P_{L} / 2$ \\ $\mathrm{P}_{\mathrm{L}}$ : e- e+ pair \\ momentum
}

Momentum distribution of electron- positron annihilated pairs

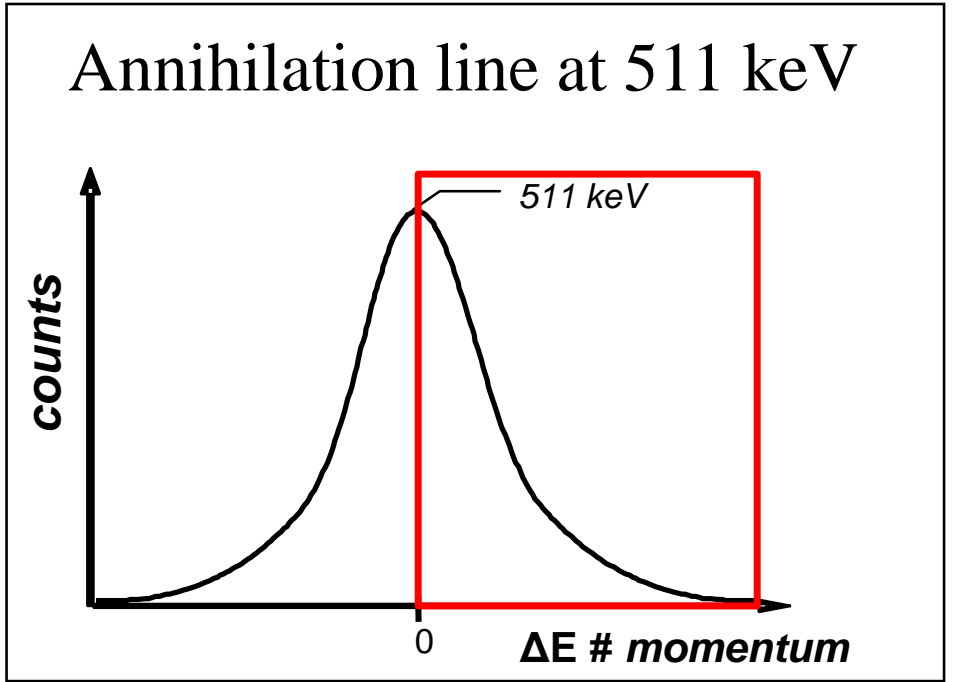


Comparison of theoretical and experimental annihilation probability densities

\section{valence}

electrons $3 \mathrm{~s}$ and $3 p$ dominate

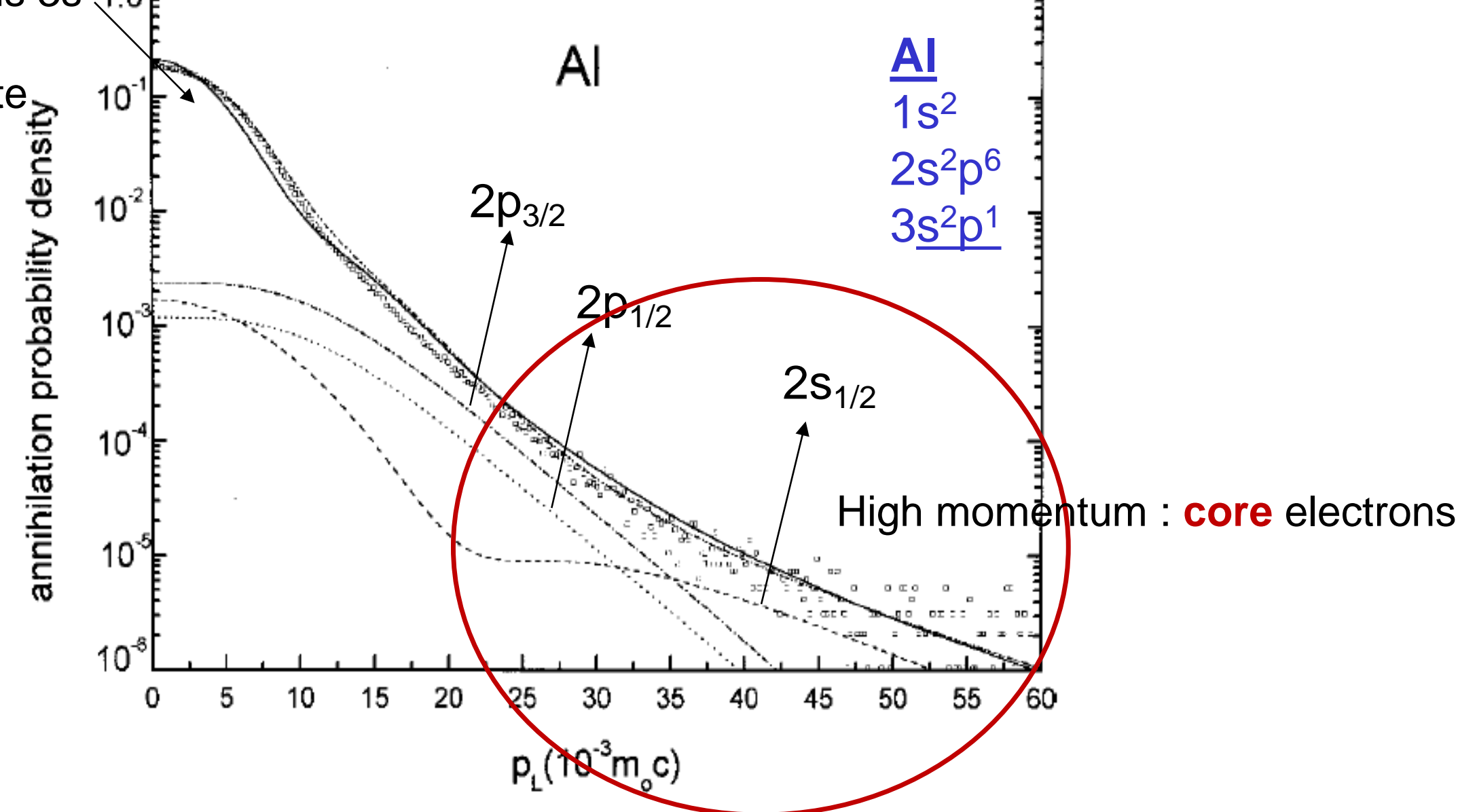




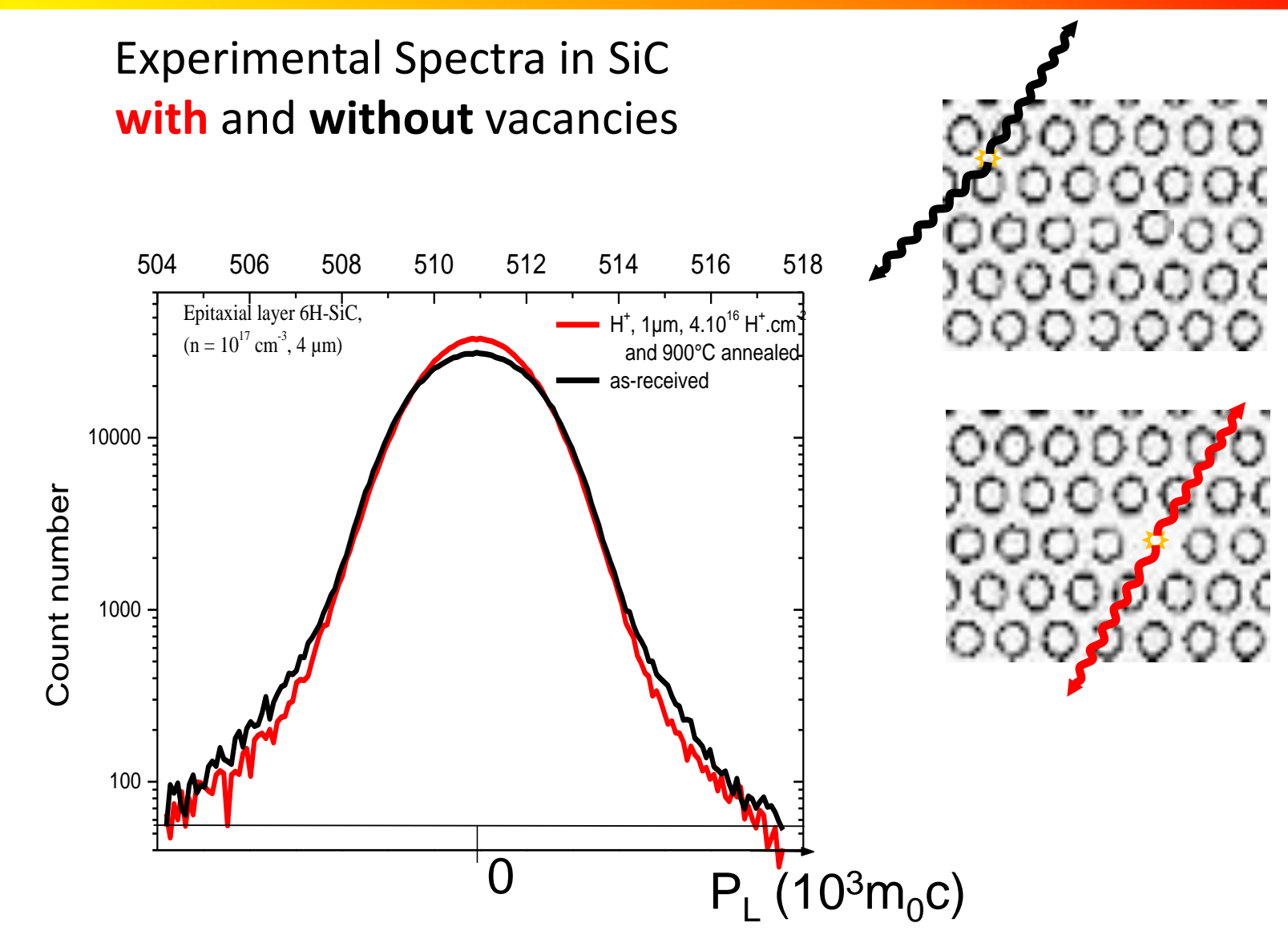

$$
\mathrm{S}_{\mathrm{L}}, \mathrm{W}_{\mathrm{L}}
$$

Annihilation characteristics in Lattice (without defects) 
Experimental Spectra in SiC with and without vacancies

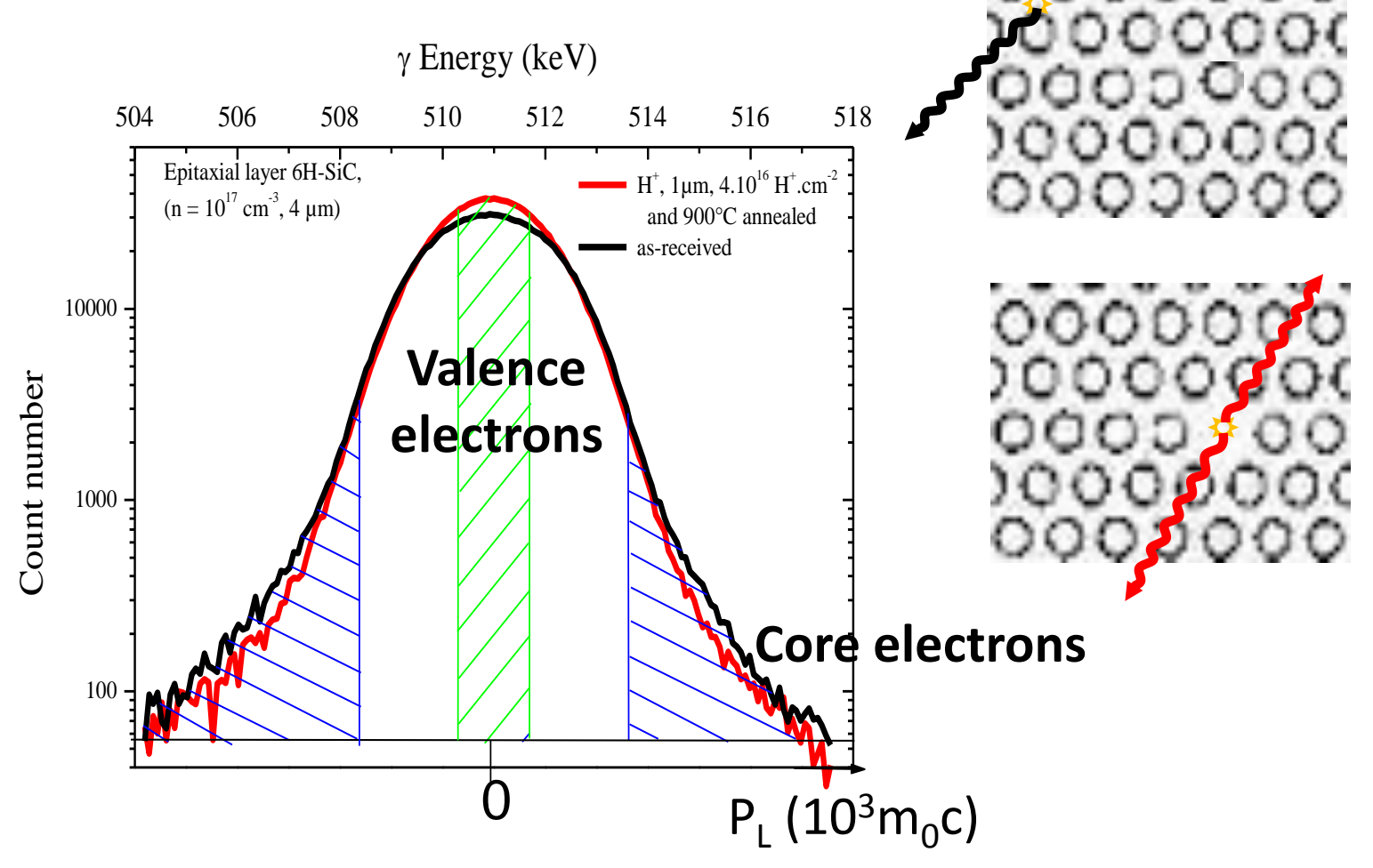

$$
\mathrm{S}_{\mathrm{L}}, \mathrm{W}_{\mathrm{L}}
$$

Annihilation characteristics in Lattice (without defects)

\section{Vacancies}

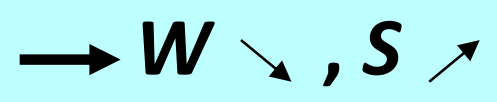

$S=$ annihilation fraction of e- e+ with low momentum, $S=A_{S} / A_{T}$

$W=$ annihilation fraction of e- e+ with high momentum,

$$
W=W_{L}+W_{R}=\left(A_{W L}+A_{W R}\right) / A_{T}
$$


Experimental Spectra in SiC with and without vacancies

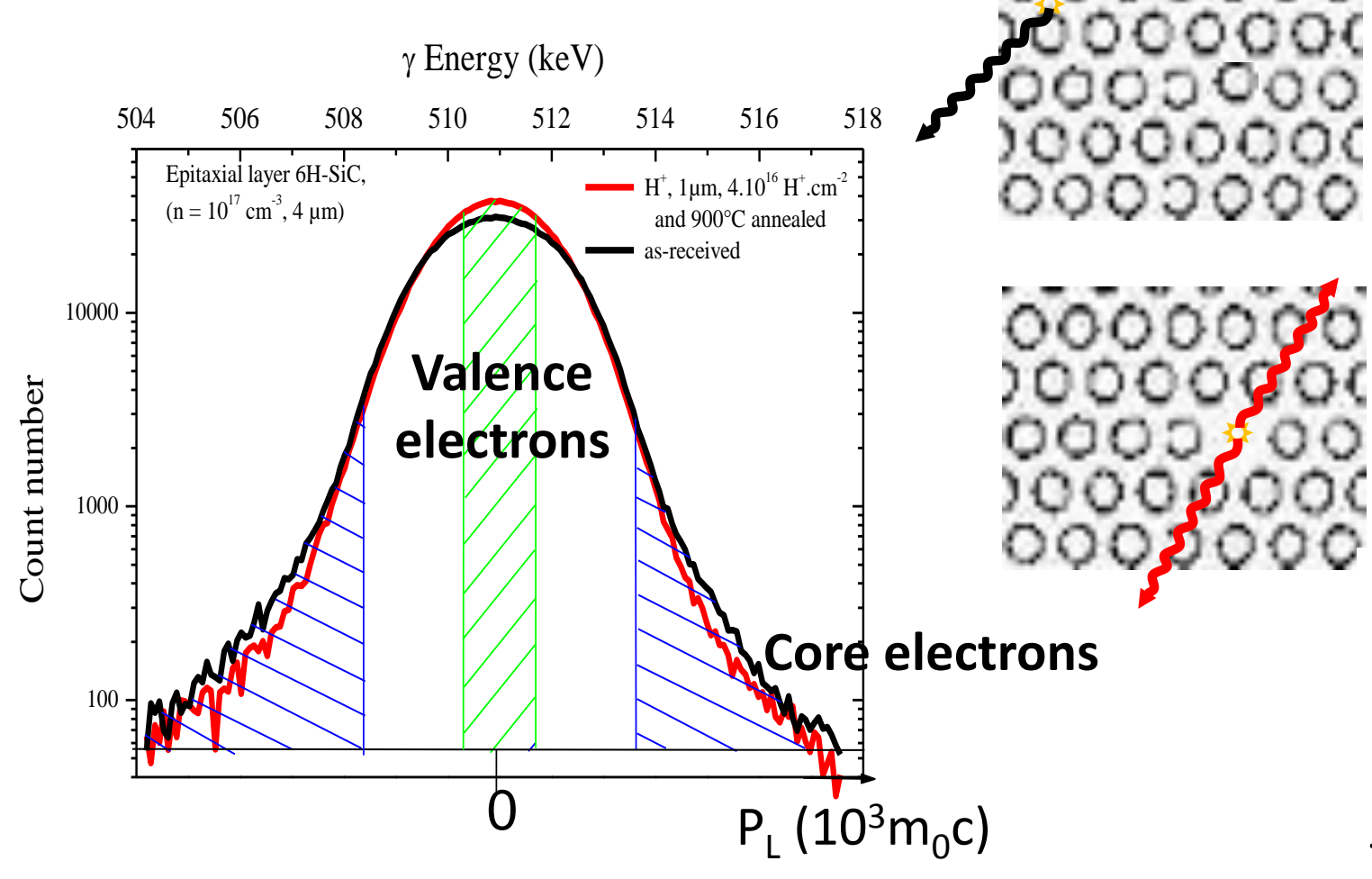

$S=$ annihilation fraction of e- e+ with low momentum, $S=A_{S} / A_{T}$ $W=$ annihilation fraction of e- e+ with high momentum,

$$
W=W_{L}+W_{R}=\left(A_{W L}+A_{W R}\right) / A_{T}
$$

$$
\mathrm{S}_{\mathrm{L}}, \mathrm{W}_{\mathrm{L}}
$$

Annihilation characteristics in Lattice (without defects)

\section{Vacancies

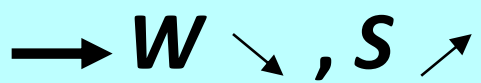

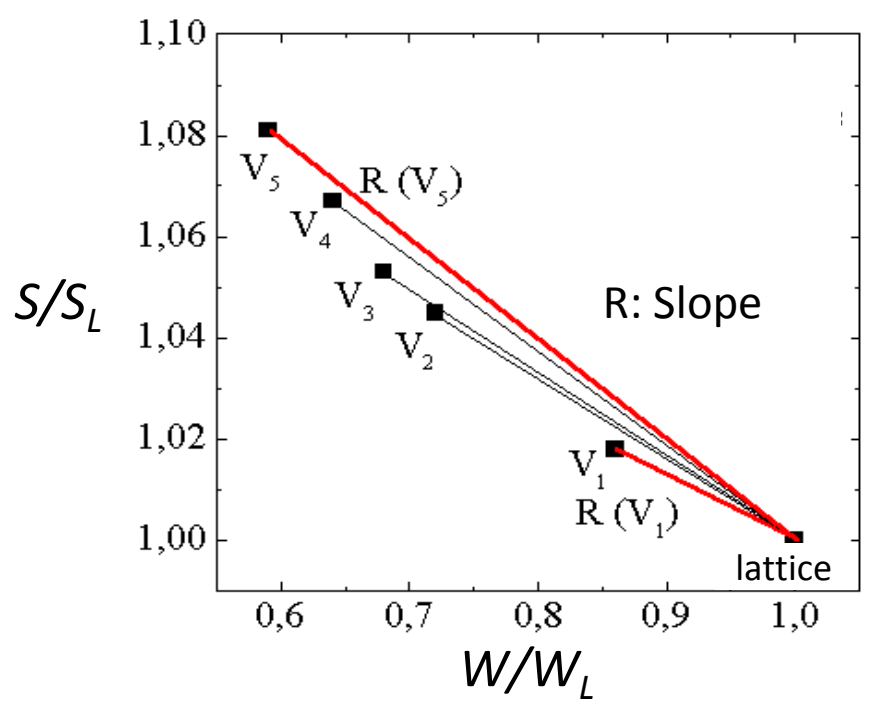




\section{Positron lifetime in W}
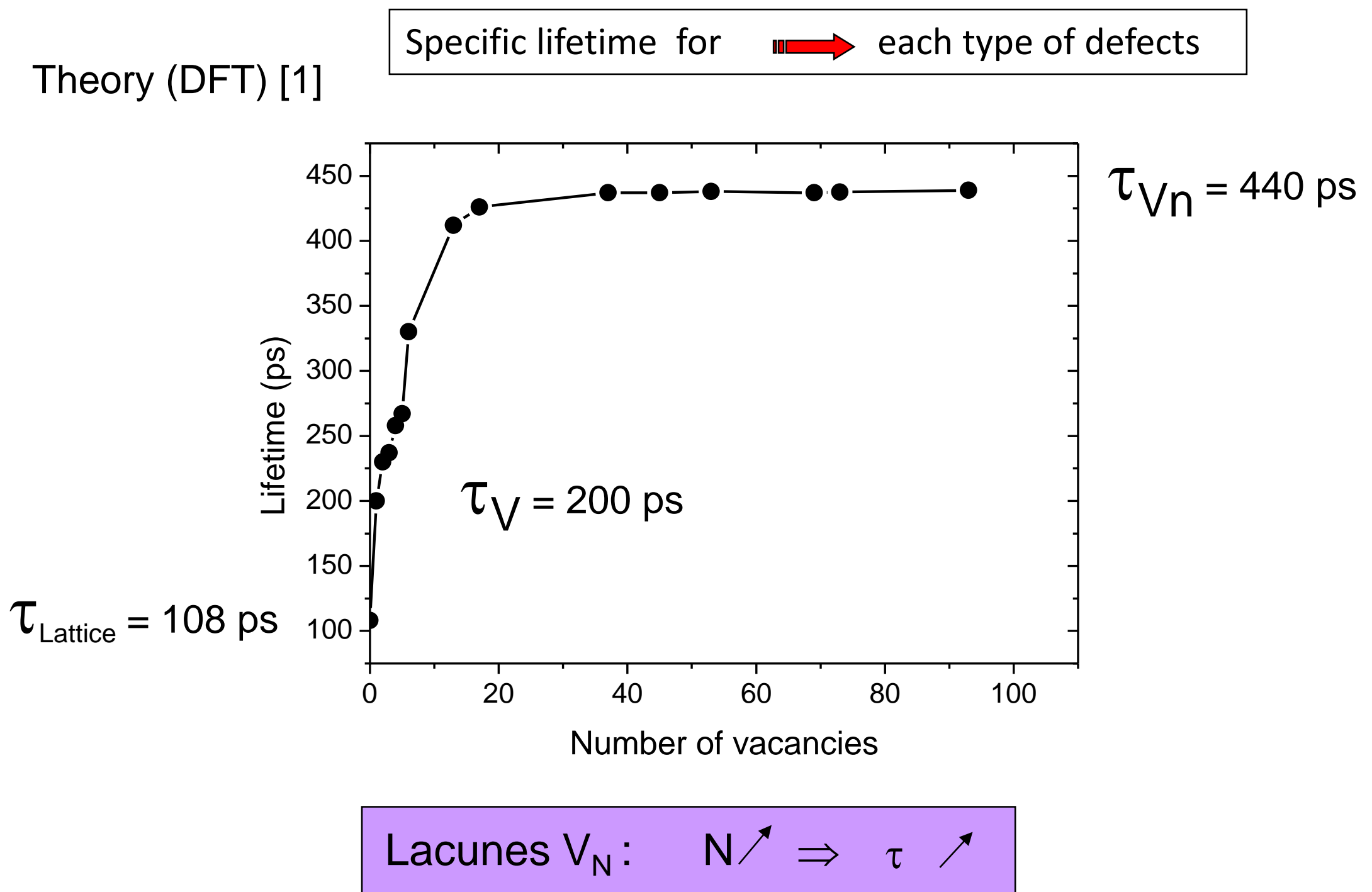
Use separate effects experiments combining irradiations in different conditions and PAS (and other techniques) measurements and calculations to characterize vacancy defects in materials:

$\checkmark$ Nature

$\checkmark$ Interactions with impurities .....

$\checkmark$ Migration

\section{In $\mathrm{W}$ and $\mathrm{UO}_{2}$}




\section{Vacancy defects in W : Single vacancies properties and V-clusters}


$\checkmark$ ITER, DEMO : steps in the development of fusion energy production

$\checkmark$ Fusion reaction ${ }^{2} \mathrm{H}+{ }^{3} \mathrm{H} \rightarrow{ }^{4} \mathrm{He}(3,5 \mathrm{MeV})+\mathbf{n}(14.1 \mathrm{MeV})$

$\checkmark$ W divertor, first walls?

dpa : displacements per atom
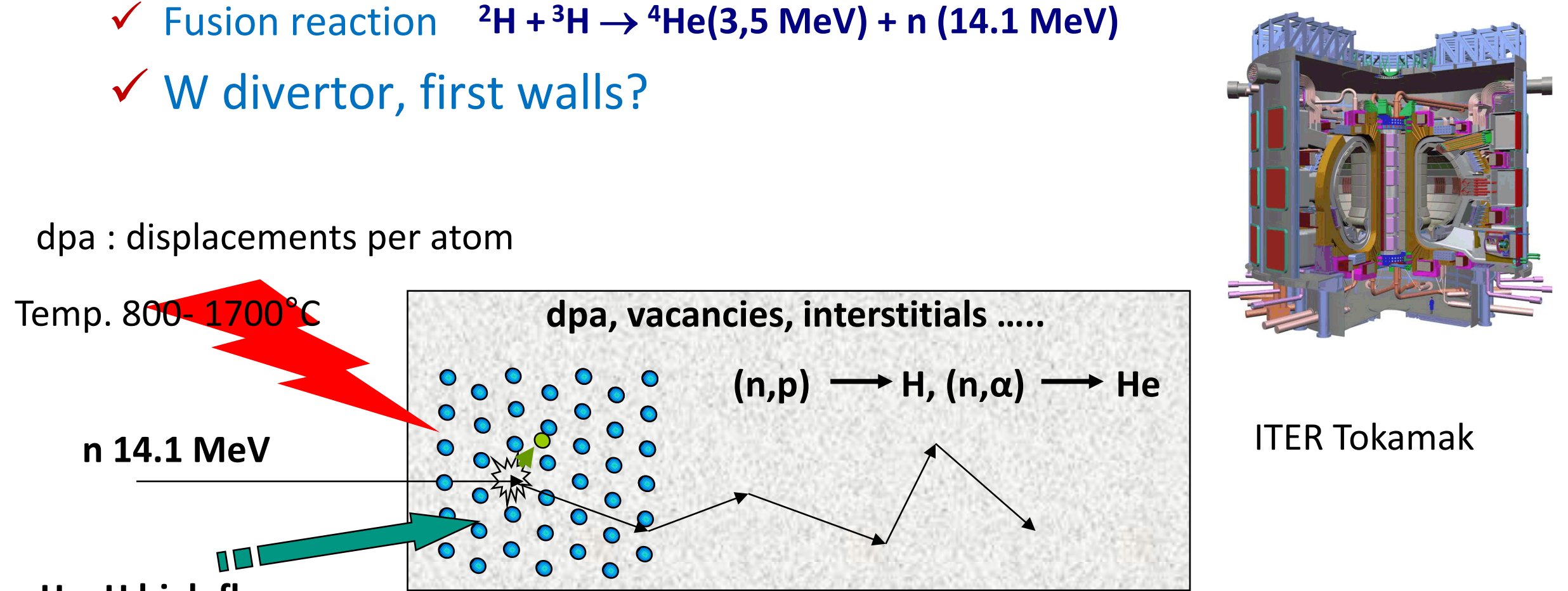

He, $\mathrm{H}$ high flux

Low energy

Change the microstructure and chemical composition

Evolution of thermal, electrical, mechanical properties of materials 


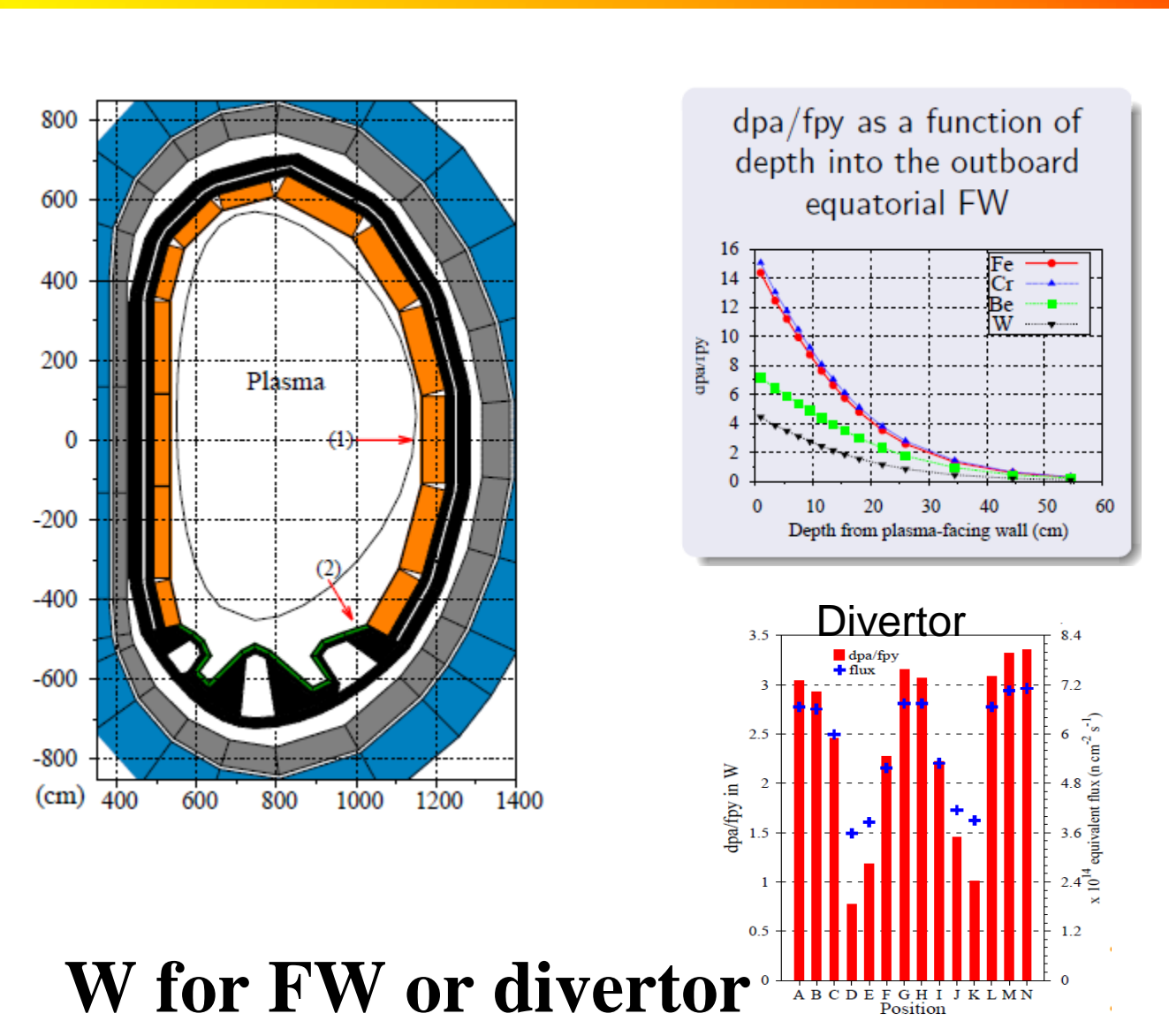

Damage dose max in 3 years $=12 \mathrm{dpa}$

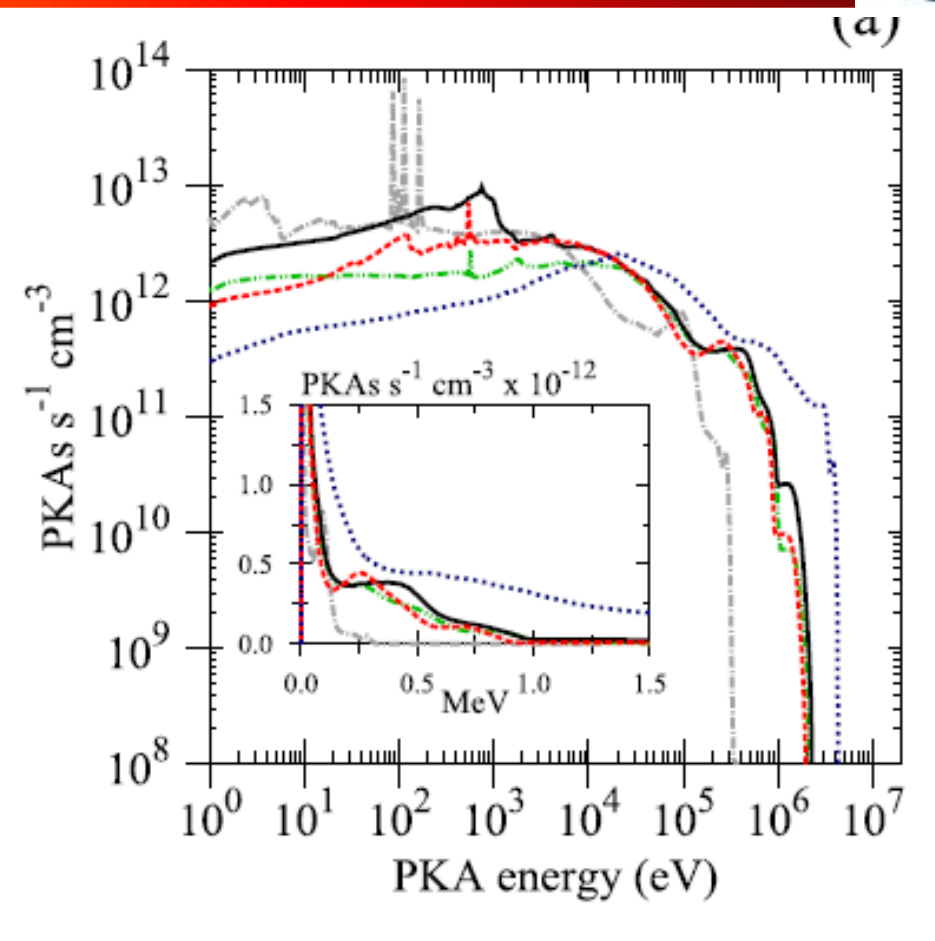

$--\mathbf{W}=$ predominant recoil

- Large energy distribution (up to $300 \mathrm{keV}$ ) mean value at $3.3 \mathrm{keV}$

[1] M.R. Gilbert et al. / Journal of Nuclear Materials 442 (2013) S755-S760,

\section{CCFE} MCNP M.R. Gilbert et al. / Journal of Nuclear Materials 467 (2015) 121 


\section{Macroscopic properties:Irradiations with neutrons}

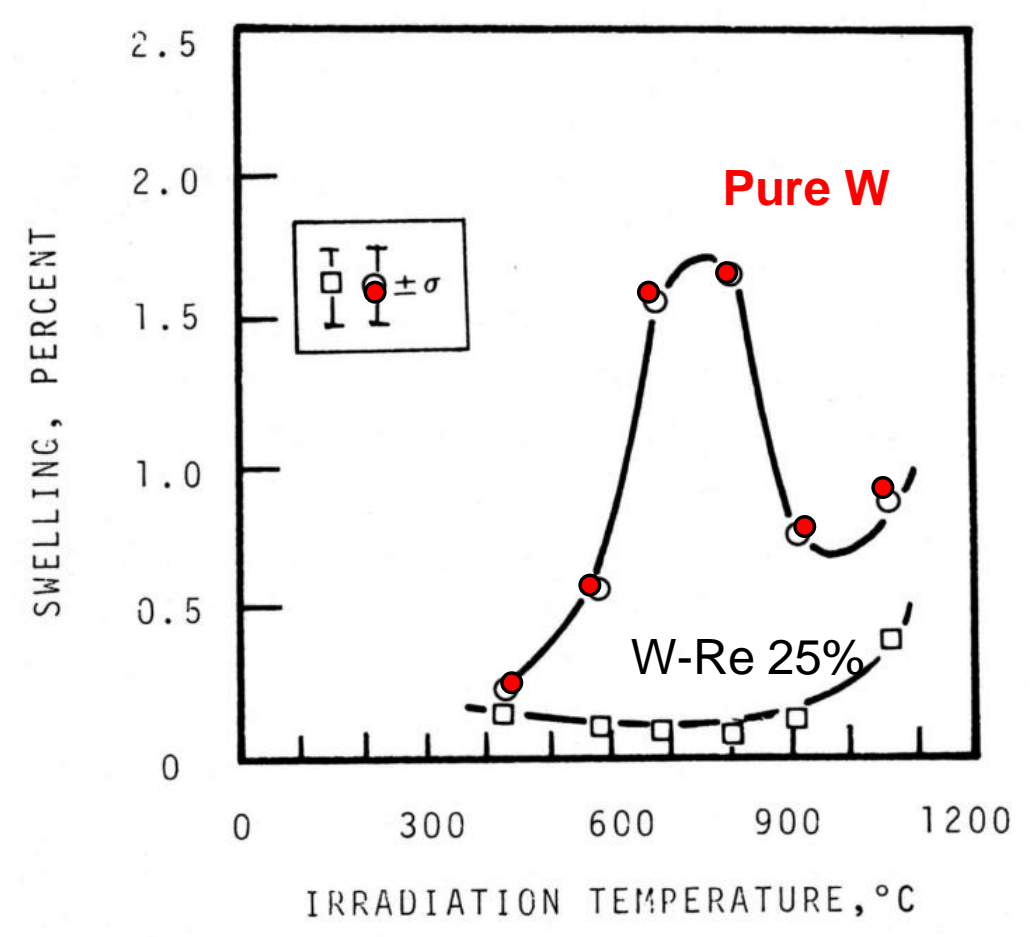

Vacancy defects?

$>$ Swelling in W irradiated with

neutrons $(E>0.1 \mathrm{MeV}), 5.5 \times 10^{26} \mathrm{n} \cdot \mathrm{m}^{2}$

(9.5dpa) [1]

[1] J.Motolich et al., Scripta Metallurgica, 8 (1974) 837-842; [2] V. Barabash, G. Federici, M. Rödig, L.L. Snead, C.H. Wu, Journal of Nuclear Materials, 283-287, Part 1 (2000) 138-146. 
Virgin samples : thin foils $(150 \mu \mathrm{m}), 7^{*} 7 \mathrm{~mm}^{2}$, annealed at $1600^{\circ} \mathrm{C} / 1 \mathrm{~h} /$ Vacuum

Implantation to induce defects

${ }^{3} \mathrm{He} 800 \mathrm{keV}$

Fluence de $10^{13}$ à $5.10^{16} \mathrm{~cm}^{-2}$

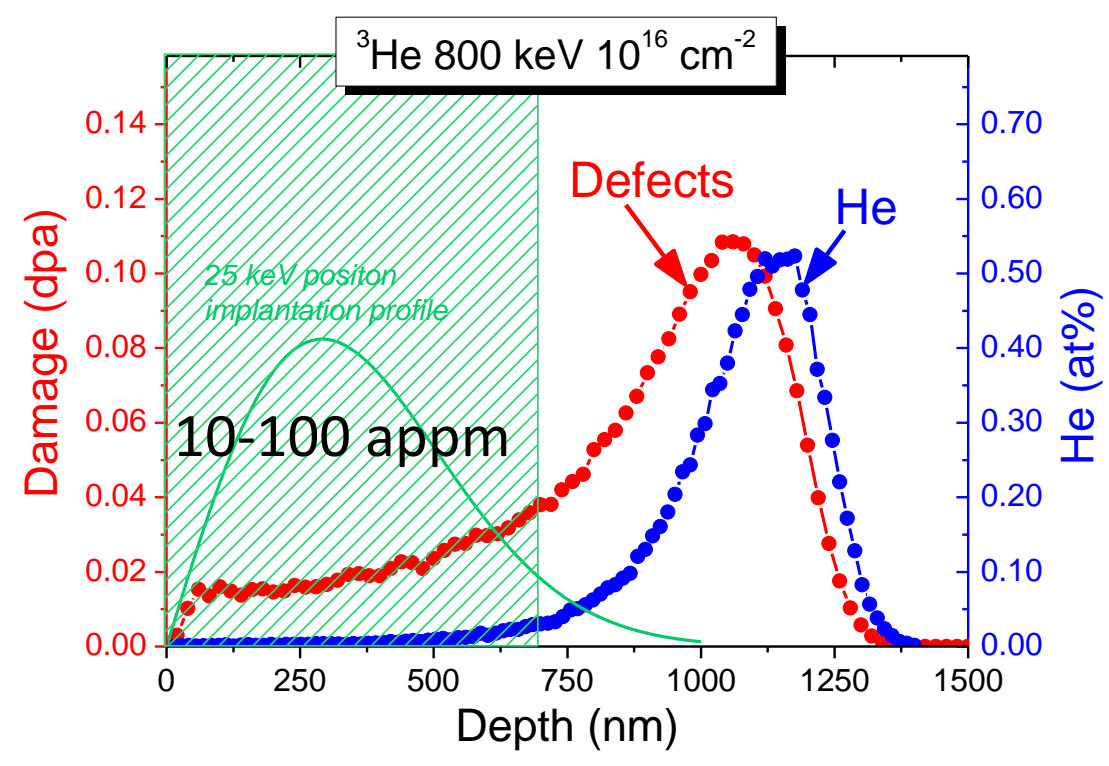

Study of defects in the

Track region with PAS

\begin{tabular}{|c|c|}
\hline Fluence & $\begin{array}{c}\text { Estimated Damage (dpa) }{ }^{[\mathrm{SRIM}]} \\
0 \text { to } 700 \mathrm{~nm}\end{array}$ \\
\hline $10^{14}$ & $2.5 \times 10^{-4}$ \\
\hline $10^{15}$ & $2.5 \times 10^{-3}$ \\
\hline $10^{16}$ & 0.025 \\
\hline $5 \times 10^{16}$ & 0.13 \\
\hline
\end{tabular}

PAS ; Positron Annihilation Spectroscopy 


\section{Implantation-induced defects}

Virgin sample annealed $1600^{\circ} \mathrm{C} / 1 \mathrm{~h} /$ vacuum

${ }^{3} \mathrm{He} 800 \mathrm{keV}$ implantation, Fluence from $10^{13}$ to $5 \times 10^{16} \mathrm{~cm}^{-2}$ (VDG Orléans)

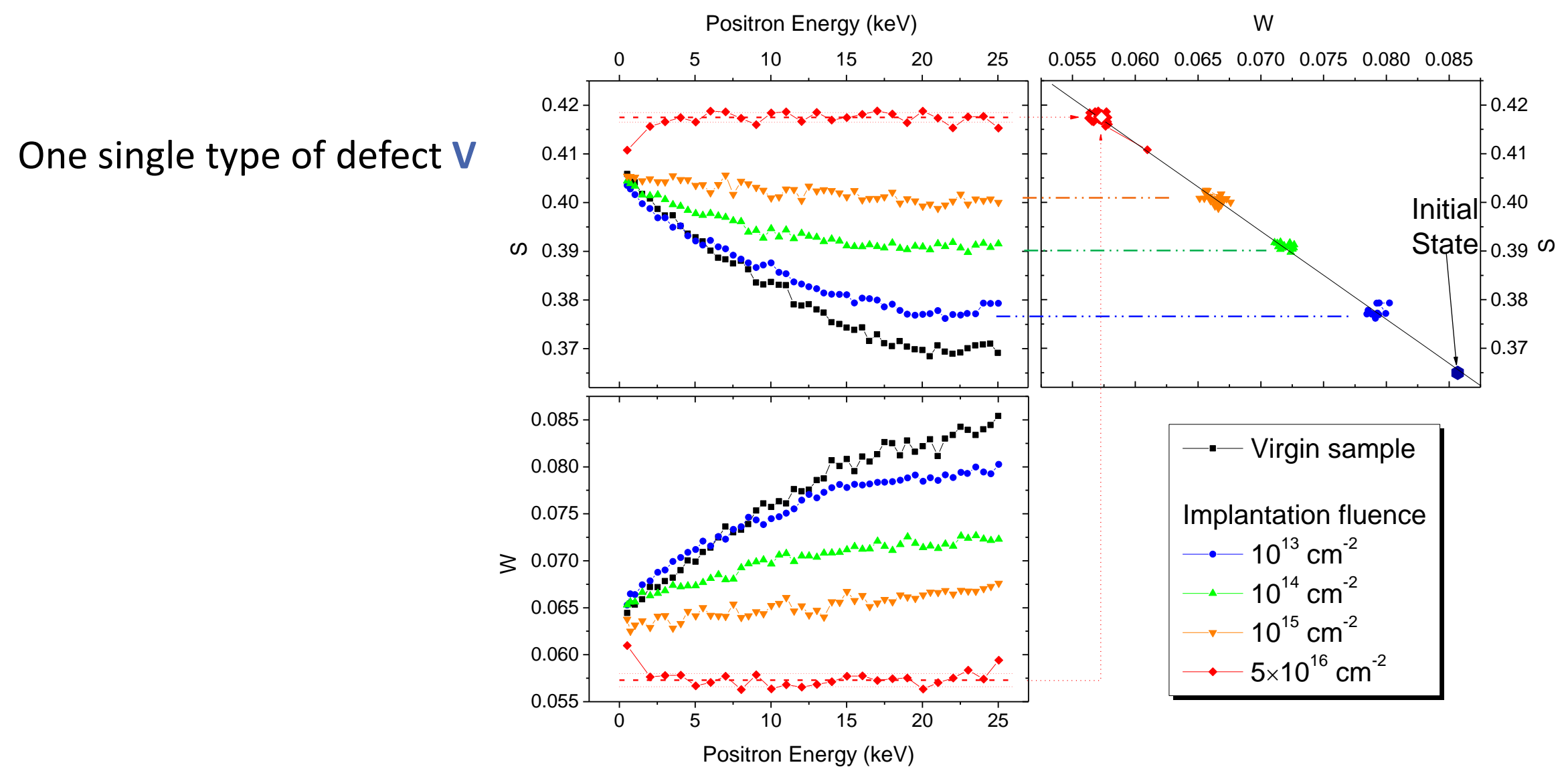

A Debelle , MF Barthe et al., J. Nucl. Mat., 376 (2008) 216-221 


\section{Single vacancy in $\mathrm{W}$}

Virgin sample : annealed $1500^{\circ} \mathrm{C} / 1 \mathrm{~h} / \mathrm{ArH}_{2}$ ${ }^{3} \mathrm{He} 800 \mathrm{keV}$ irradiation

Fluence from $10^{14}$ to $5 \times 10^{16} \mathrm{~cm}^{-2}$

$\Rightarrow$ One single type of defect

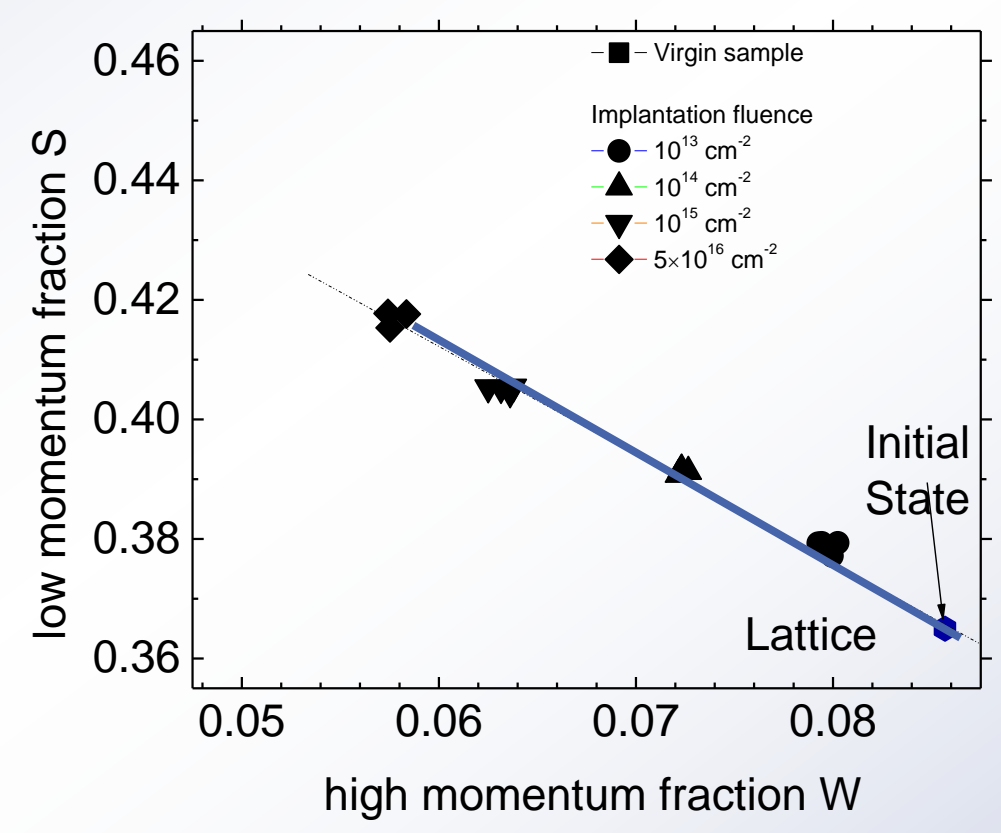

[1] A Debelle , MF Barthe et al, JNM.376 (2008) 216; [2] T. Troev, E. Popov, et al, NIMB 267, (2009), 335; [3] P.E. Lhuillier, MF Barthe et al., PSS ,C6, (2009), 2329,

[4] C.Becquart,et al JNM 403, Issues 1-3, 2010, 75-88 


\section{Single vacancy in $\mathrm{W}$}

Virgin sample : annealed $1500^{\circ} \mathrm{C} / 1 \mathrm{~h} / \mathrm{ArH}_{2}$ ${ }^{3} \mathrm{He} 800 \mathrm{keV}$ irradiation

Fluence from $10^{14}$ to $5 \times 10^{16} \mathrm{~cm}^{-2}$

$\Rightarrow$ One single type of defect

Positron Lifetime In agreement with calculations $\tau=200 \pm 0.4 p s \quad I=98 \pm 0.2 \%,[3] \quad \tau_{\text {VCalc }}=200 p s$ [2]

\section{Single vacancy (V)}

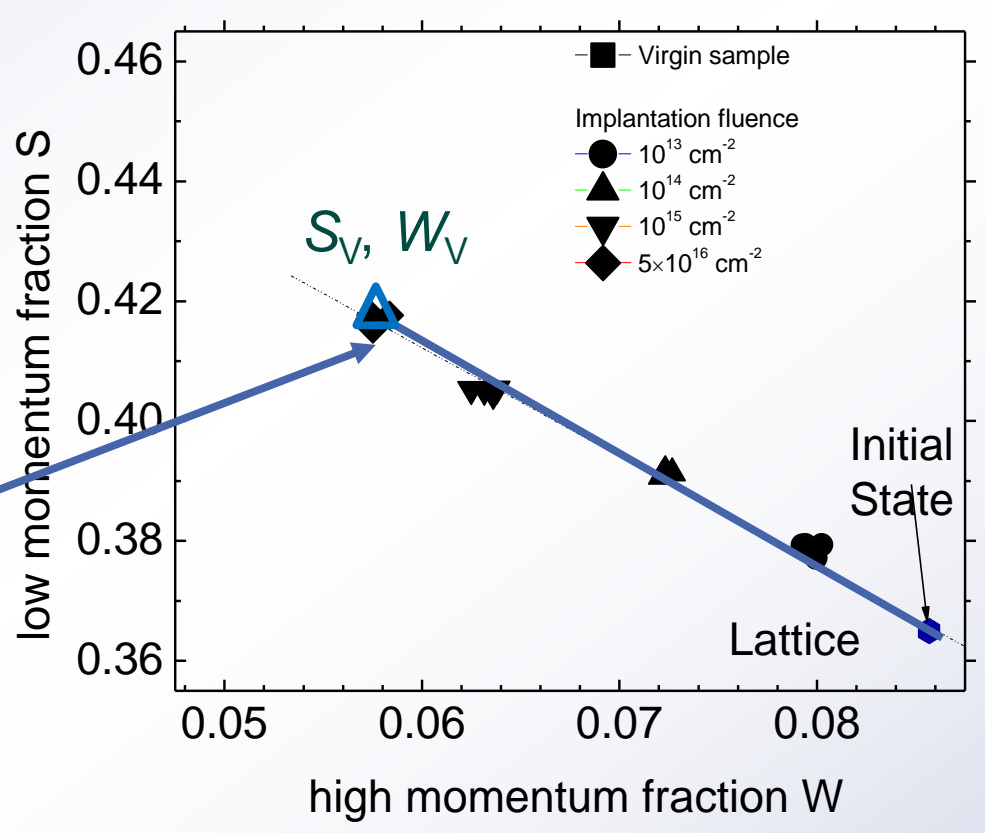

[1] A Debelle , MF Barthe et al, JNM.376 (2008) 216; [2] T. Troev, E. Popov, et al, NIMB 267, (2009), 335; [3] P.E. Lhuillier, MF Barthe et al., PSS ,C6, (2009), 2329,

[4] C.Becquart,et al JNM 403, Issues 1-3, 2010, 75-88 


\section{Single vacancy in $\mathrm{W}$}

Virgin sample : annealed $1500^{\circ} \mathrm{C} / 1 \mathrm{~h} / \mathrm{ArH}_{2}$ ${ }^{3} \mathrm{He} 800 \mathrm{keV}$ irradiation

Fluence from $10^{14}$ to $5 \times 10^{16} \mathrm{~cm}^{-2}$

Trapping in one trap $=2$ annihilation states

- Lattice, $S_{L}$ and $\tau_{L}=110 p s$

- Single Vacancy $V, S_{V}$
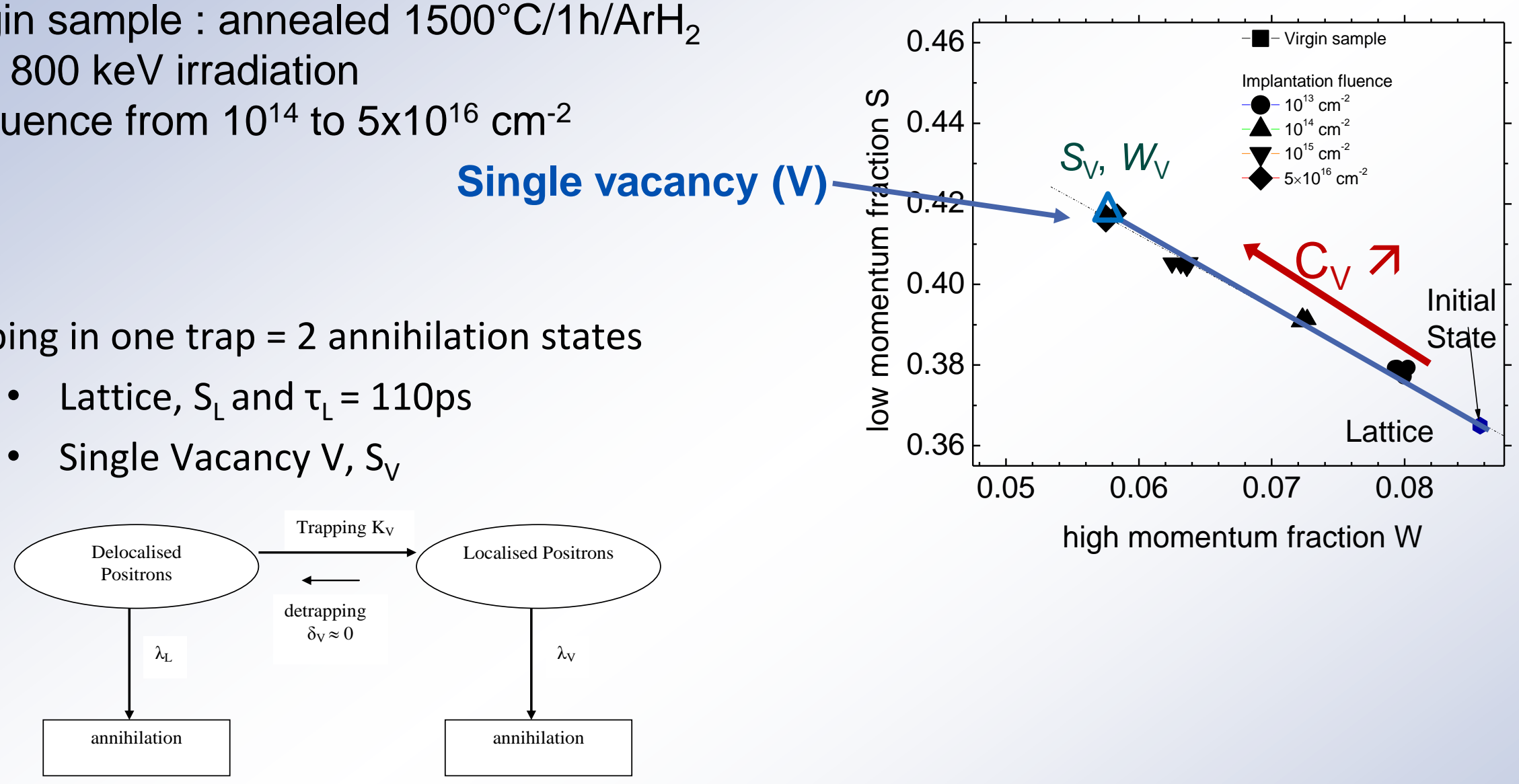

high momentum fraction $\mathrm{W}$

$$
C_{V}=\frac{\lambda_{L}}{\mu_{V}} \frac{A_{\text {norm }}}{1-A_{\text {norm }}}
$$

$$
A_{\text {norm }}=S_{\text {norm }}, S_{\text {norm }}=S / S_{L}
$$

[1] PE Lhuillier Thesis CEMHTI 2010, 


\section{$800 \mathrm{keV}^{3} \mathrm{He}$ irradiated W}

PAS Experimental data

SRIM

Marlowe

Trapping in one trap $=2$ annihilation states

- Lattice, $\mathrm{S}_{\mathrm{L}}$ and $\tau_{\mathrm{L}}=110 \mathrm{ps}$

- Single Vacancy $\mathrm{V}, \mathrm{S}_{\mathrm{V}}$
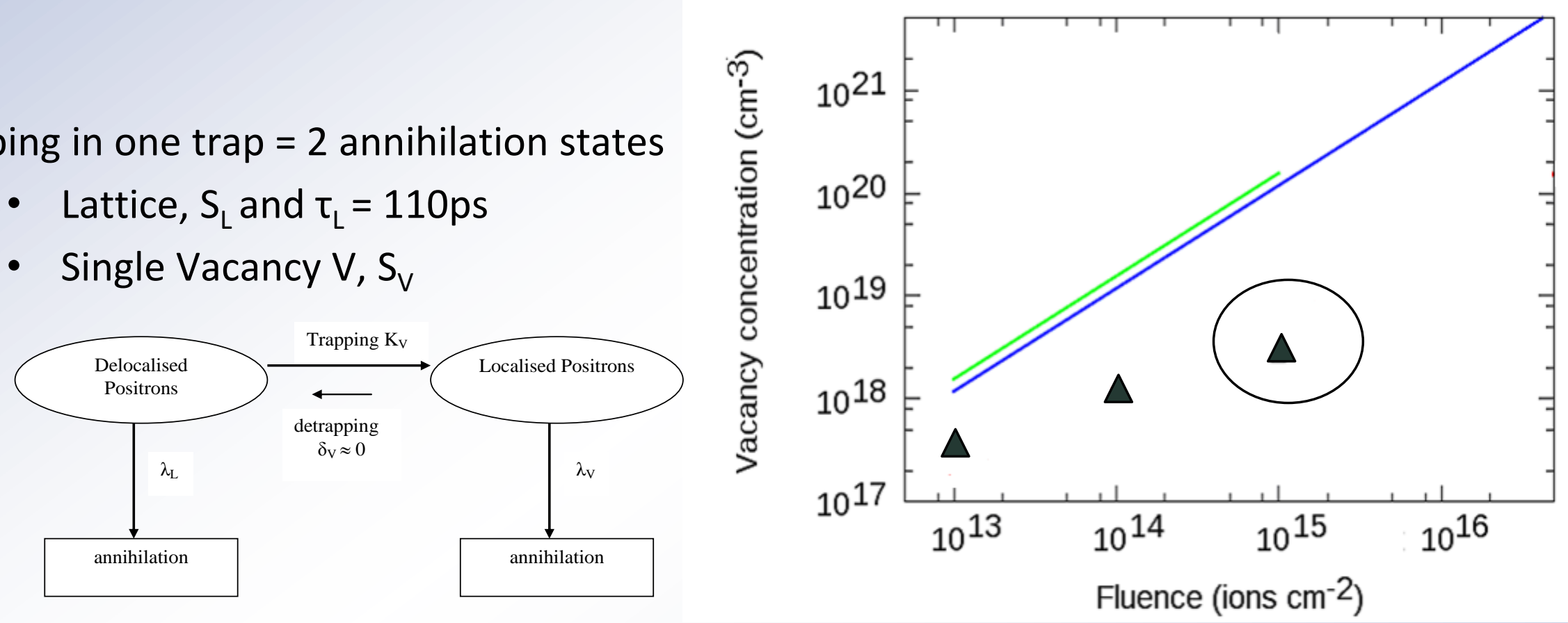

Trapping rate, $\mathrm{K}_{\mathrm{v}}=\mathrm{C}_{\mathrm{V}} \cdot \mu_{\mathrm{V}}$ $\mu_{\mathrm{V}}$ trapping specific coefficient,

$$
\mu_{\mathrm{V}=} 4 \pm 2 \times 10^{14} \mathrm{~s}^{-1}[1]
$$

$$
C_{V}=\frac{\lambda_{L}}{\mu_{V}} \frac{A_{\text {norm }}}{1-A_{\text {norm }}}
$$

$$
A_{\text {norm }}=S_{\text {norm }}, S_{\text {norm }}=S / S_{L}
$$

[1] PE Lhuillier Thesis CEMHTI 2010, [2] A De Backer, C Becquart, C. Domain, MF Barthe, JNM 429 (2012) 78-91, [3] C.Becquart,et al JNM 403, Issues $1-3,2010,75-88$ 


\section{$800 \mathrm{keV}^{3} \mathrm{He}$ irradiated W}

LAKIMOCA (C. Domain EdF) [3]

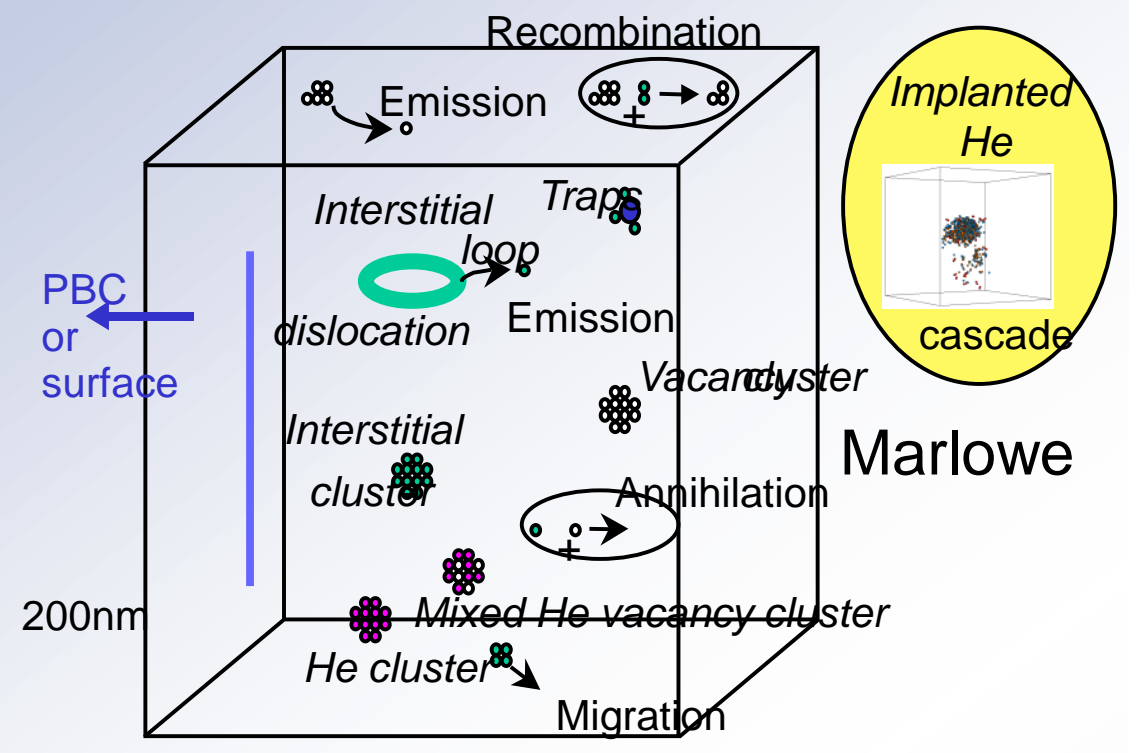

PAS Experimental data SRIM Marlowe

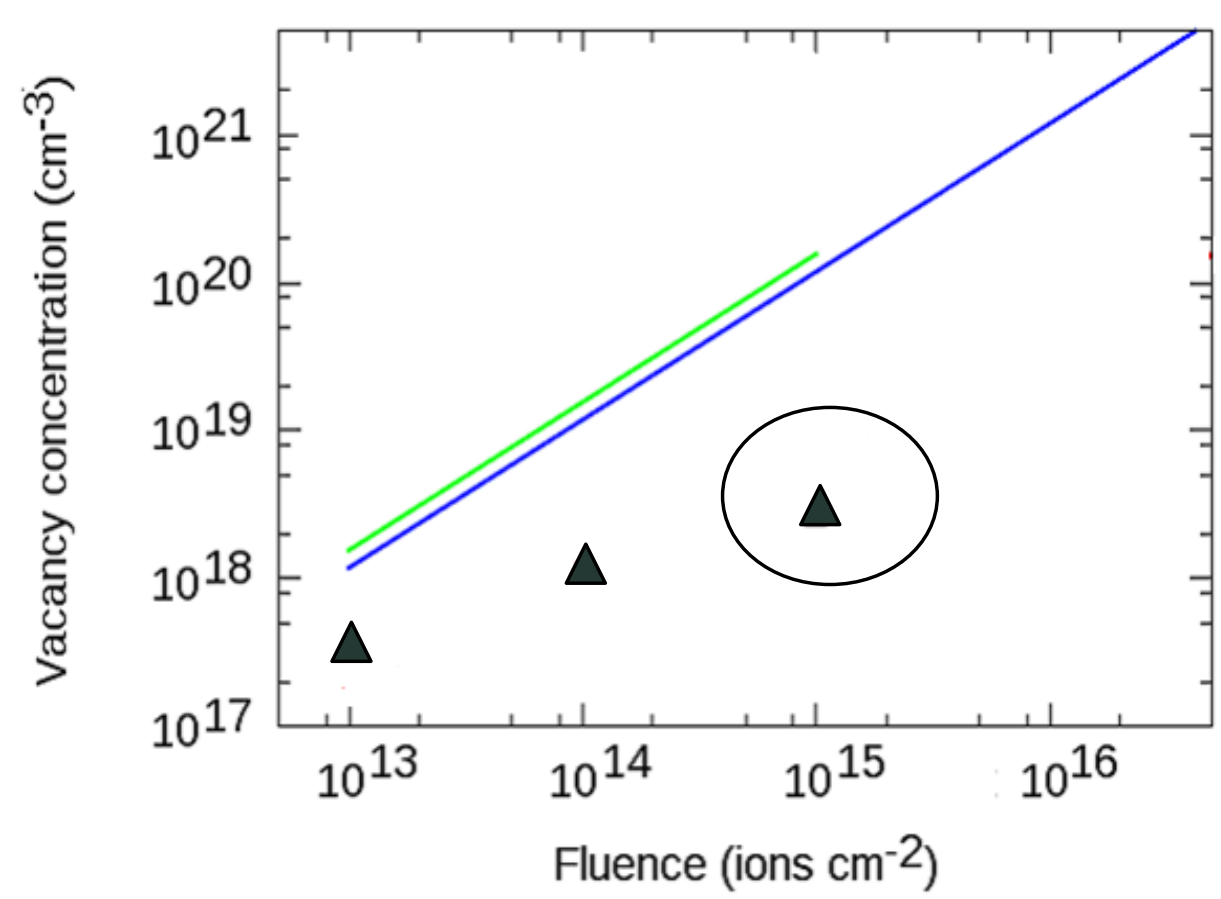

[1] PE Lhuillier Thesis CEMHTI 2010, [2] A De Backer, C Becquart, C. Domain, MF Barthe, JNM 429 (2012) 78-91, [3] C.Becquart,et al JNM 403, Issues 1-3, 2010, 75-88 
LAKIMOCA (C. Domain EdF) [3]

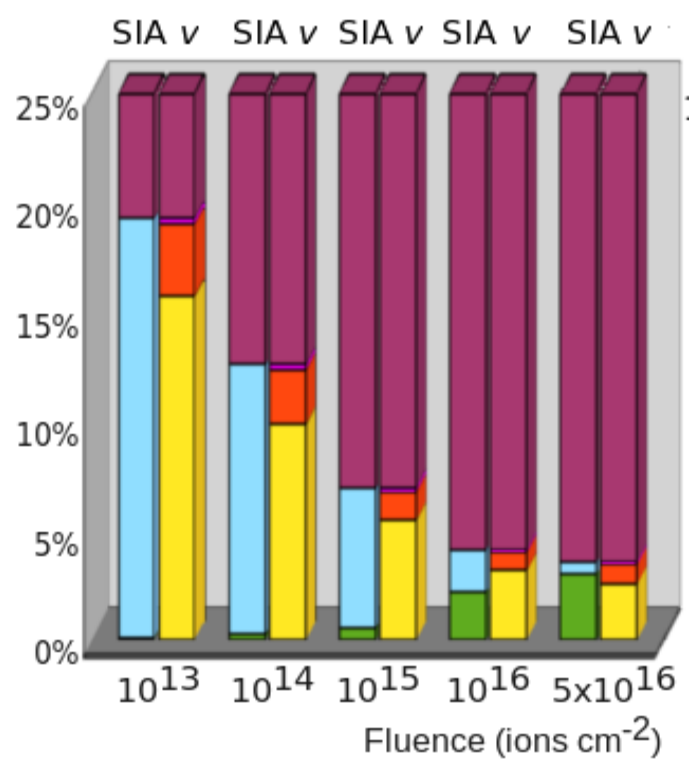

$\square$ Annihilation $\square \mathrm{mv} m>1$ $\square$ Desorption $\square v$

\begin{tabular}{c|c} 
Species & Em $(\mathrm{eV})$ \\
\hline mono-SIA (1I) & 0.013 \\
mono- $v(1 \mathrm{v})$ & 1.66
\end{tabular}

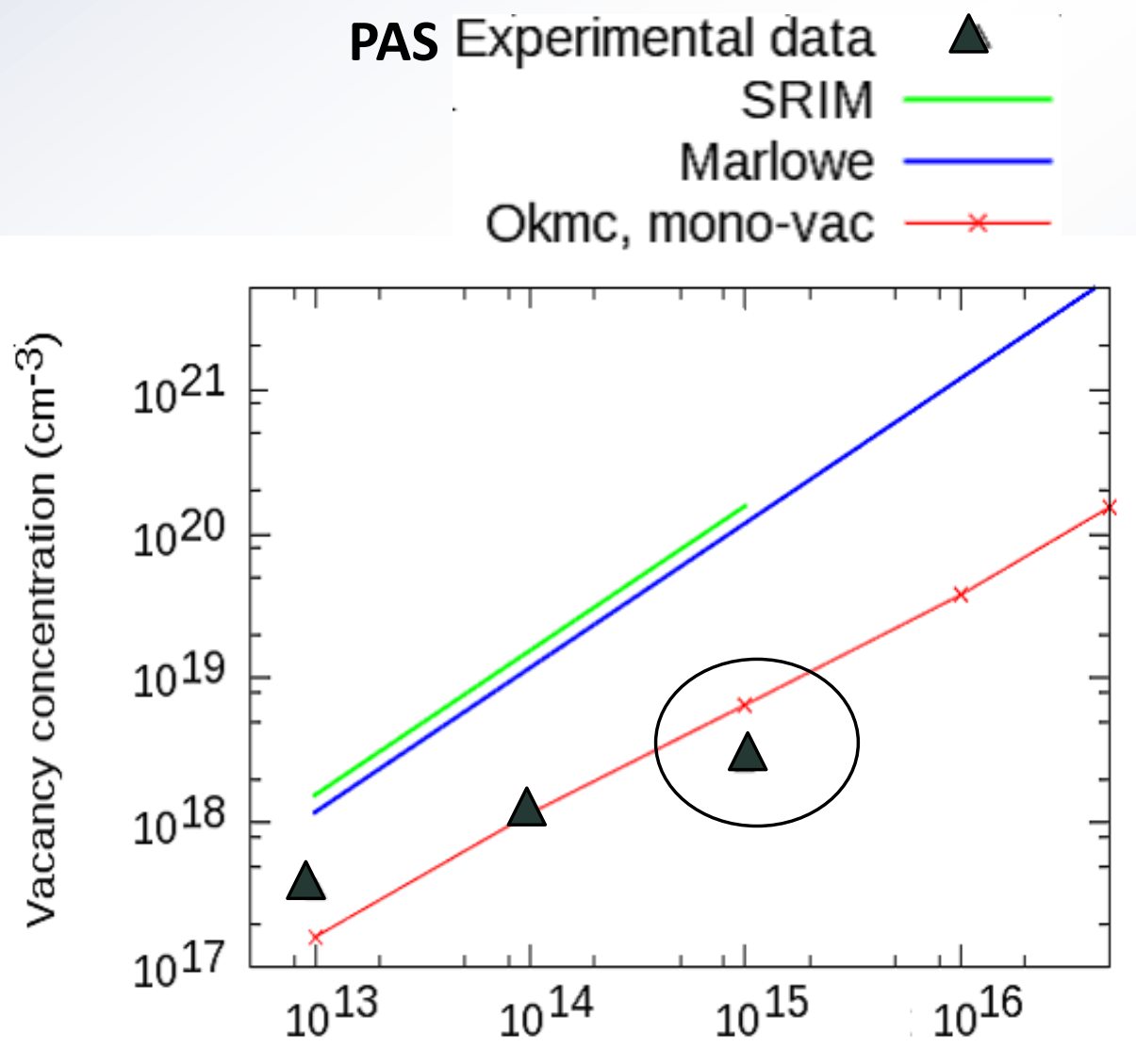

Fluence (ions $\mathrm{cm}^{-2}$ )

[1] PE Lhuillier Thesis CEMHTI 2010, [2] A De Backer, C Becquart, C. Domain, MF Barthe, JNM 429 (2012) 78-91, [3] C.Becquart,et al JNM 403, Issues 1-3, 2010, 75-88 


\section{Single vacancy/ V-clusters in $\mathrm{W}$}

Virgin sample : annealed $1500^{\circ} \mathrm{C} / 1 \mathrm{~h} / \mathrm{ArH}_{2}$ ${ }^{3} \mathrm{He} 800 \mathrm{keV}$ irradiation

Fluence from $10^{14}$ to $5 \times 10^{16} \mathrm{~cm}^{-2}$

$\square$ One single type of defect

Single vacancy (V)
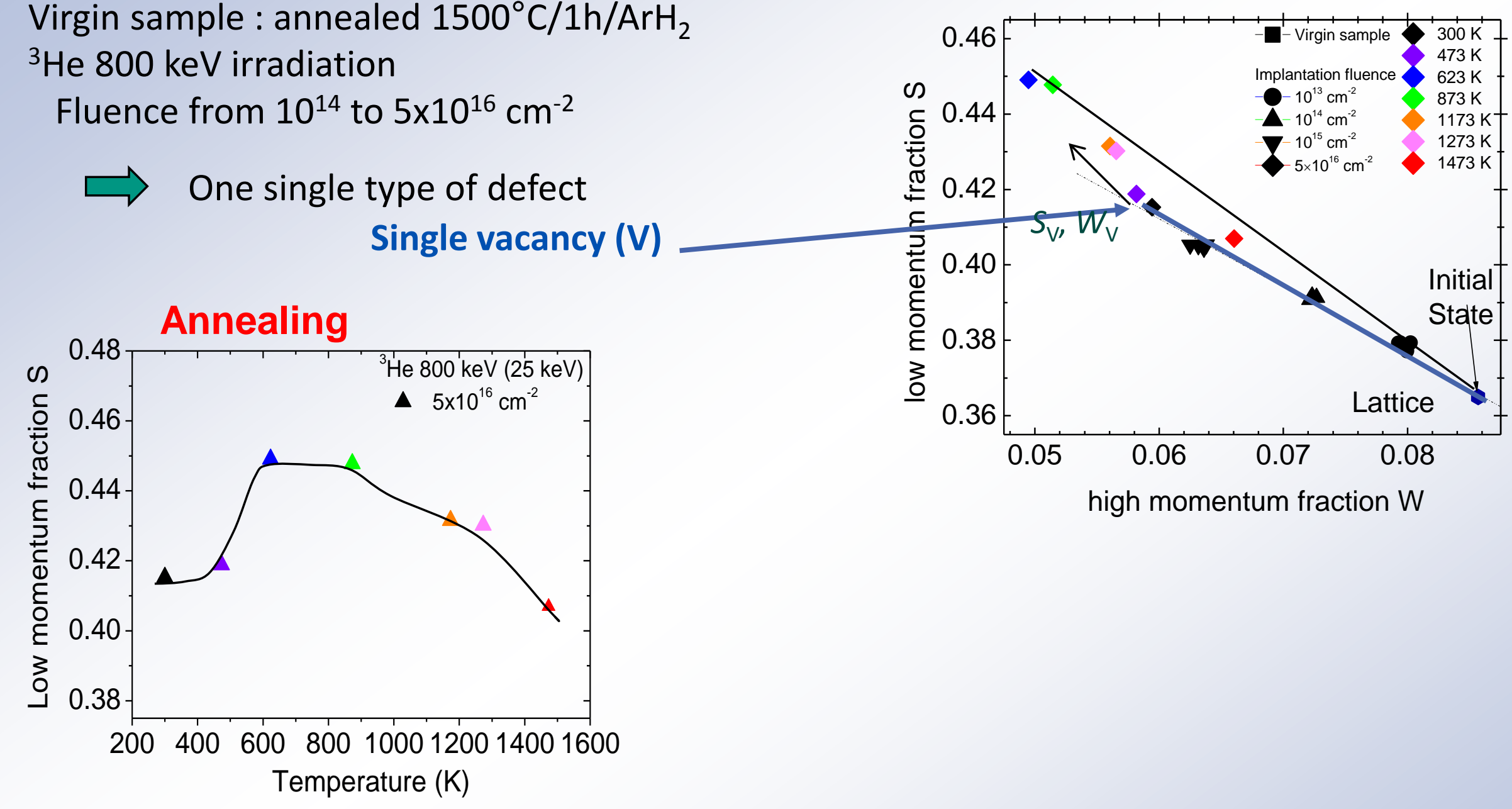

[1] A Debelle , MF Barthe et al, JNM.376 (2008) 216; [2] T. Troev, E. Popov, et al, NIMB 267, (2009), 335; [3] P.E. Lhuillier, MF Barthe et al., PSS ,C6, (2009), 2329, [4] C.Becquart,et al JNM 403, Issues 1-3, 2010, 75-88 


\title{
Vacancy defects in self ions irradiated W :
}

\author{
Positron annihilation spectroscopy \\ Transmission electron microscopy
}




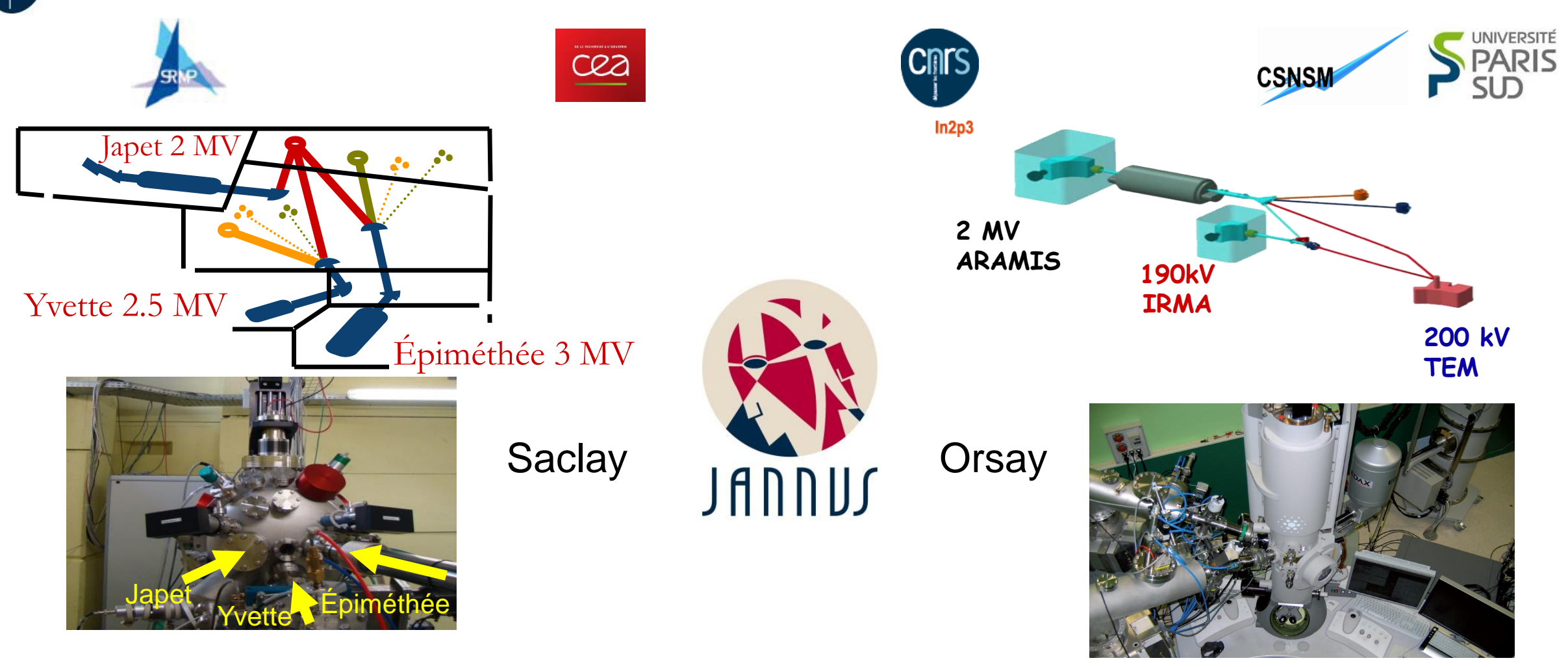

Experiments done at JANNuS (Joint Accelerators for Nanoscience and Nuclear Simulation), France

Irradiation W 20 MeV in bulk : postmortem characterizations
Irradiation W 1.2 MeV in thin foils: in situ and post- mortem characterizations

PAS, TEM

Cemhti csusw 


\section{$20 \mathrm{MeV}$ W irradiation: low dpa}
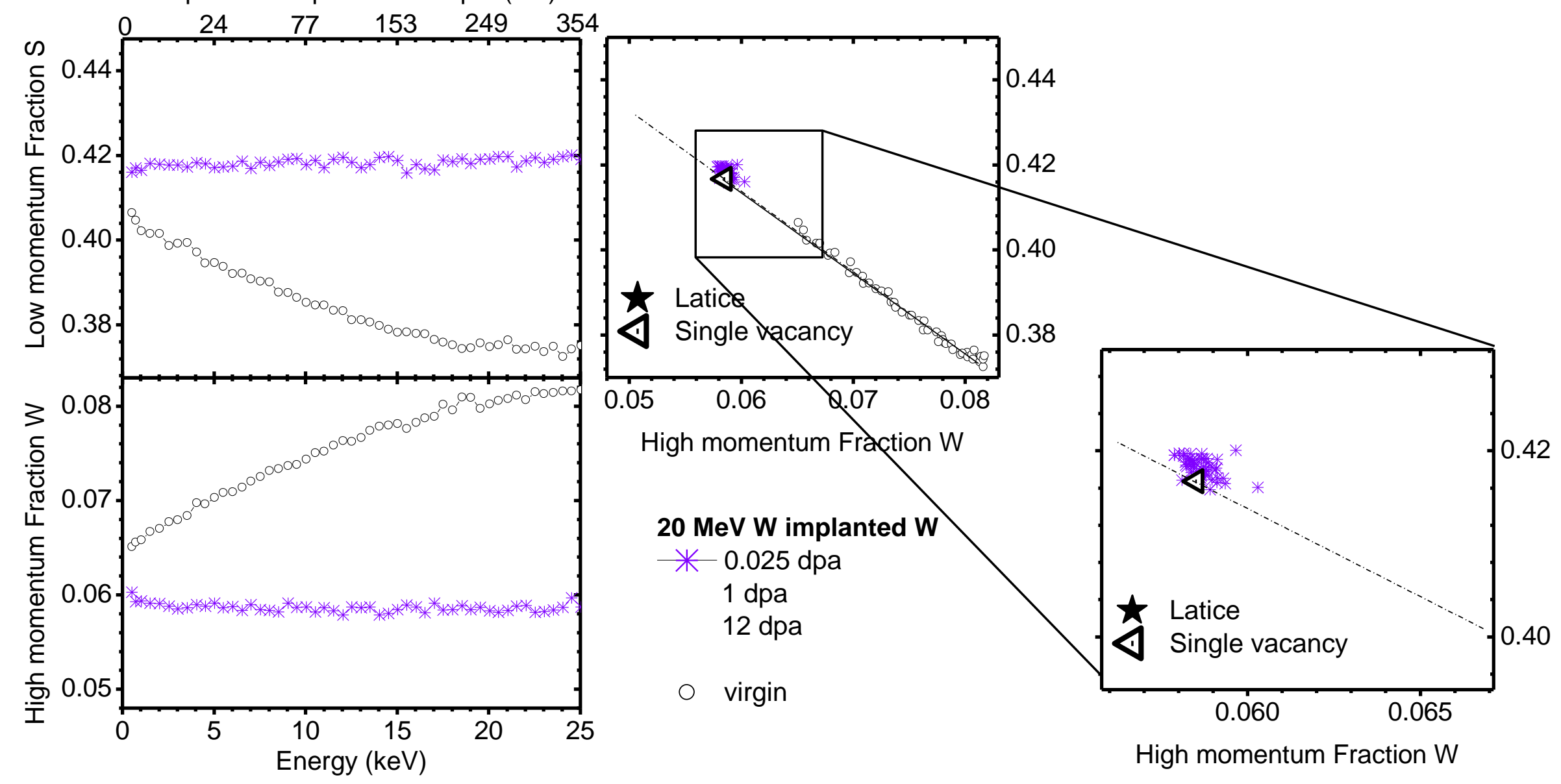

V-clusters (small 3D V-cluster+ V-loops) are created even at low dpa 


\section{$\mathrm{W}$ irradiated 1.2 MeV W ions at 0.017 dpa at $R T$}

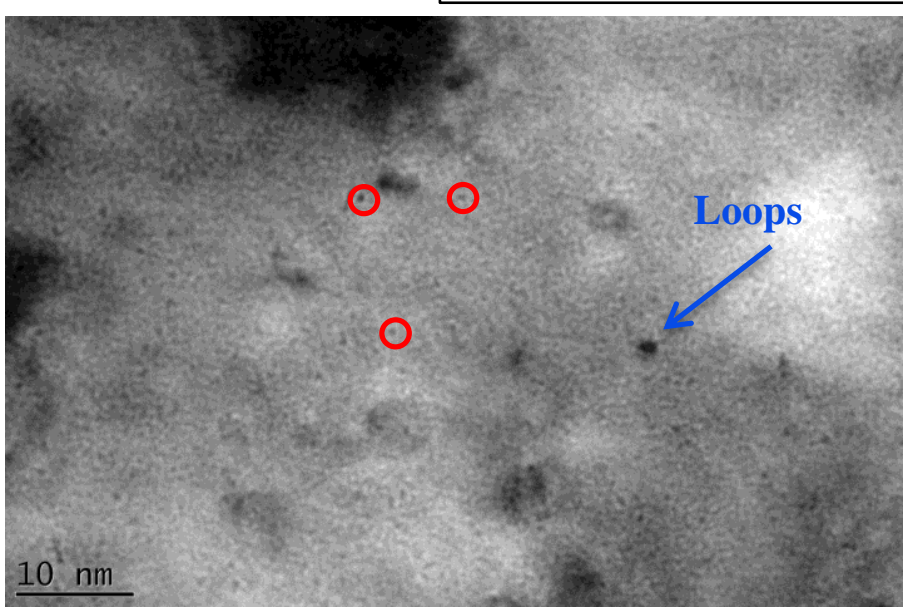

Image 1: over focused image, cavities appear black surrounded by a white halo

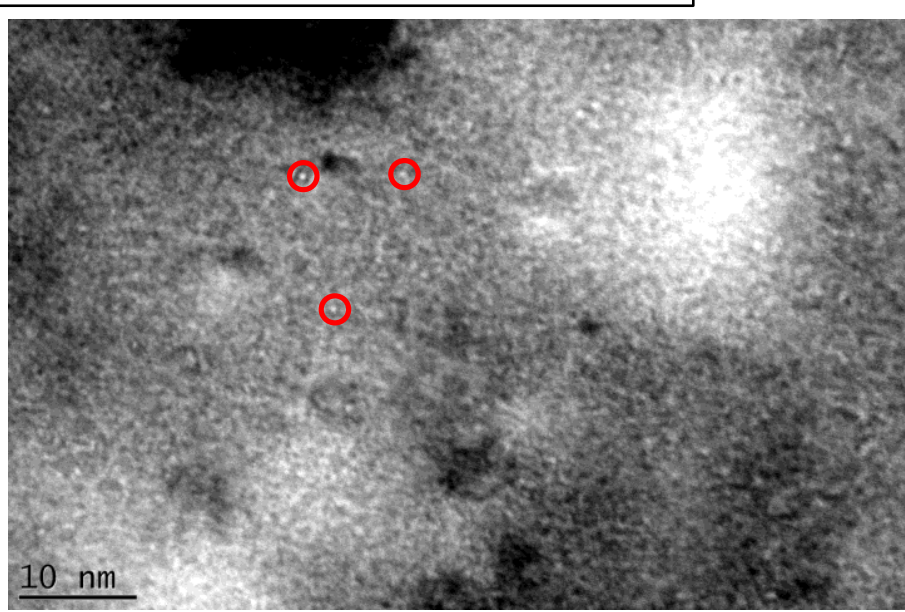

Image 2: under focused image, cavities appear white surrounded by a black halo

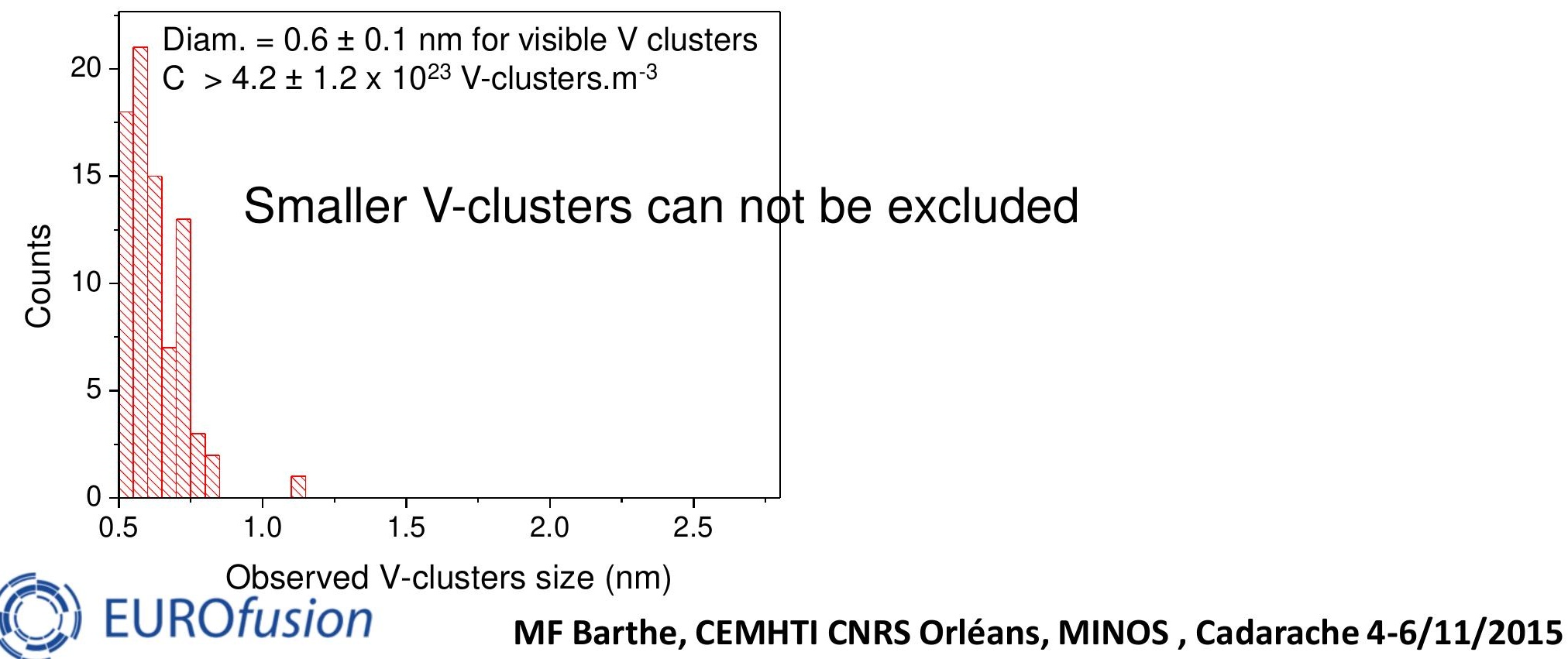




\section{$20 \mathrm{MeV}$ W irradiation: effect of dpa}

cers

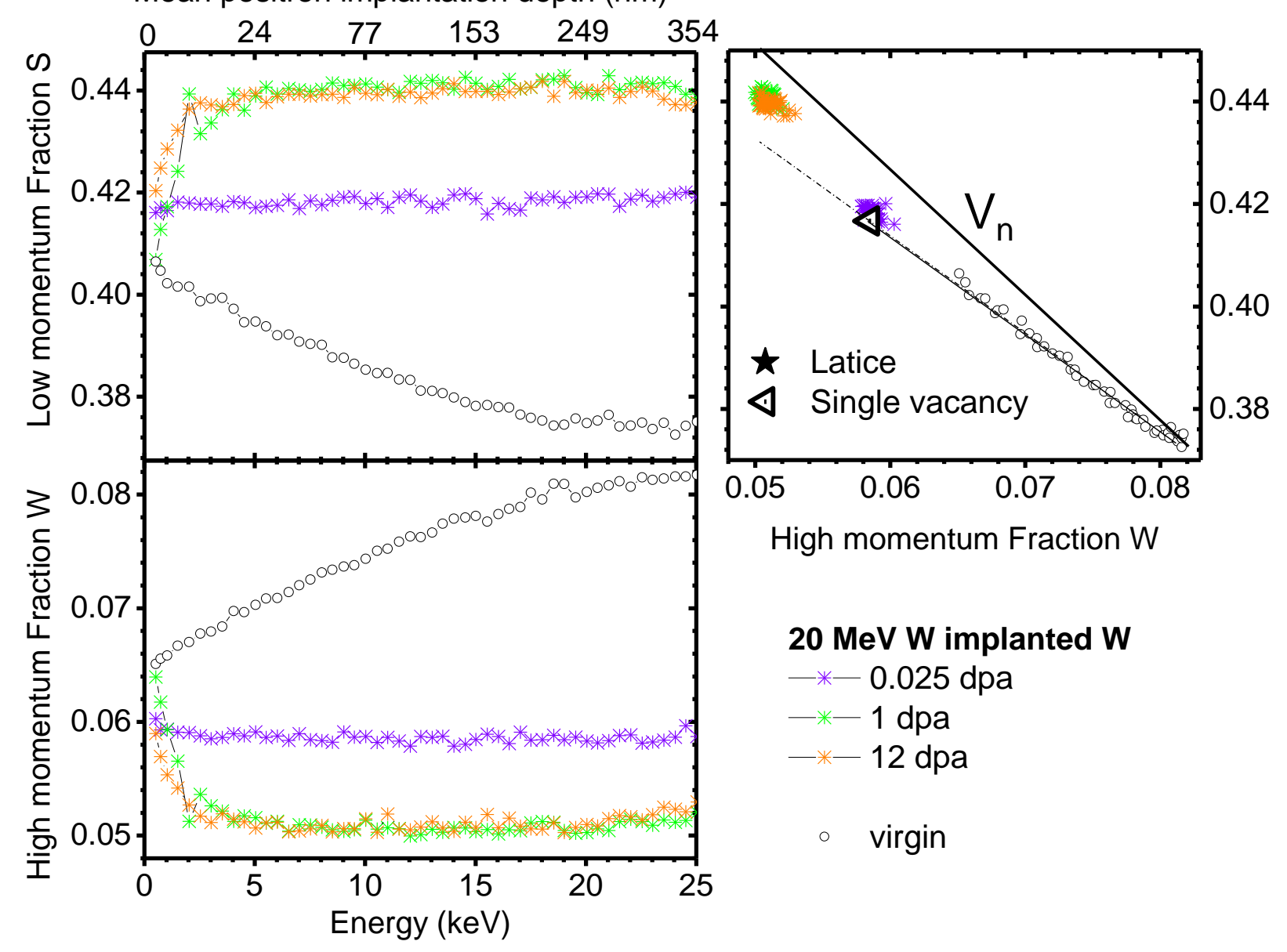

V-clusters (small 3D V-cluster+ V-loops) are created even at low dpa Size and/or concentration of vacancy clusters increases with dpa Saturation from $1 \mathrm{dpa}$ 


\section{Annealing in vacuum : effect of dpa}

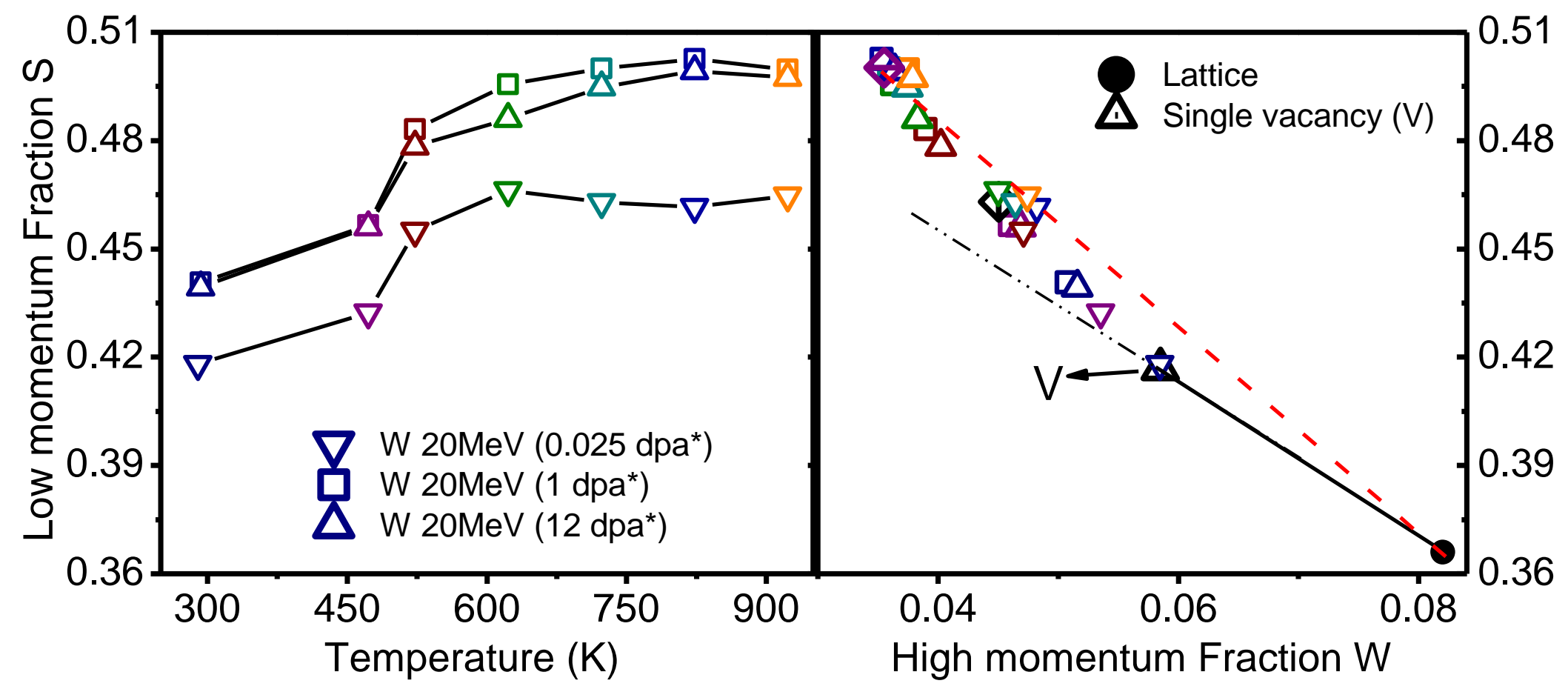




\section{Annealing in vacuum : effect of dpa}

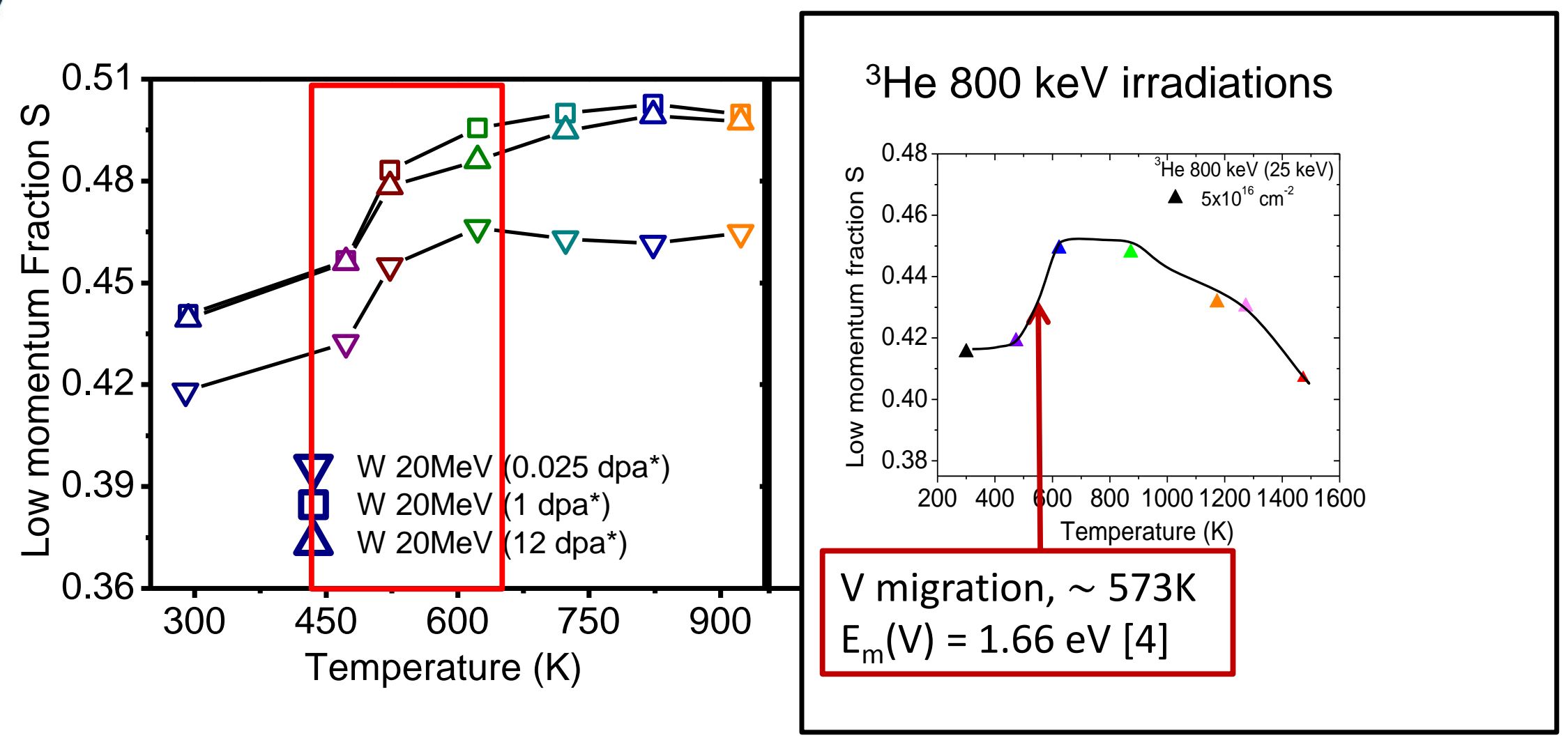

$>\forall$ dpa clustering occurs in the same temperature range as for single vacancies mainly due to $\mathrm{V}$ migration and agglomeration on small clusters 


\section{Vacancy defects in $\mathrm{UO}_{2}$ :}




\section{Experiments : Indirect detection of defects in $\mathrm{UO}_{2}$}

$\mathrm{XRD}$, lattice parameter changes, [1,2,3 ]
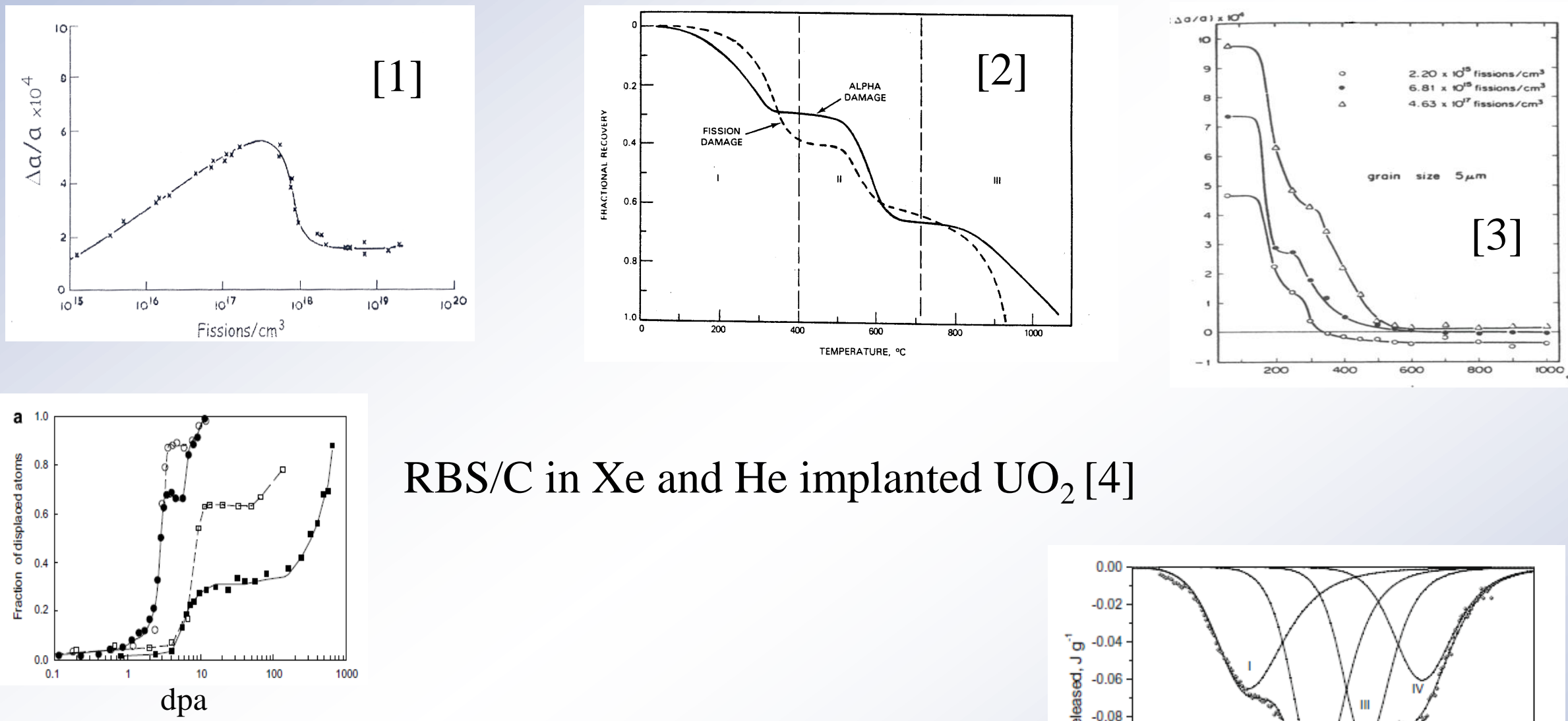

$\mathrm{RBS} / \mathrm{C}$ in $\mathrm{Xe}$ and $\mathrm{He}$ implanted $\mathrm{UO}_{2}[4]$

Thermal properties in $(\mathrm{U}, \mathrm{Pu}) \mathrm{O}_{2}$ samples, [5]

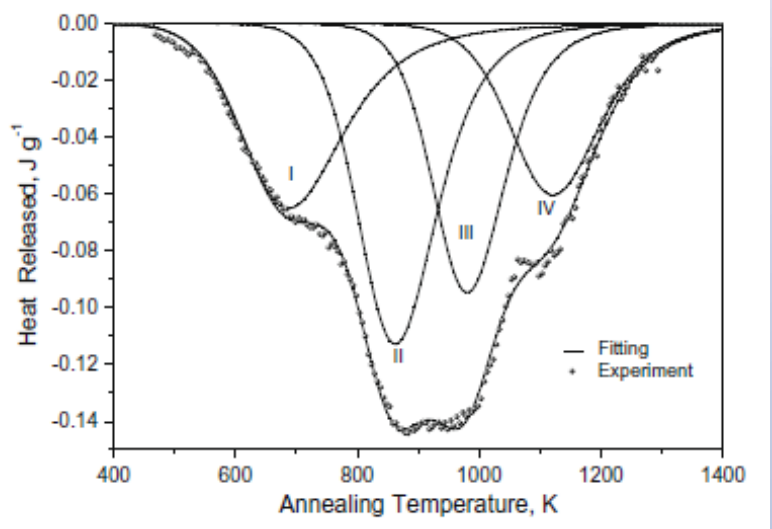

[1] J.H.Davies et al, JNM 41 (1971), 143., [2] Weber W.J, JNM 114 (1983), 213 [3] N.Nakae, JNM 80 (1979), 314-322 , [4] F. Garrido et al. NIM B 266 (2008) 2842-2847, [5] D. Staicu, T. Wiss et al. JNM 397 (2010) 8-18 


\section{Experiments : Indirect detection of defects in $\mathrm{UO}_{2}$}

XRD, lattice parameter changes, [1,2,3 ]
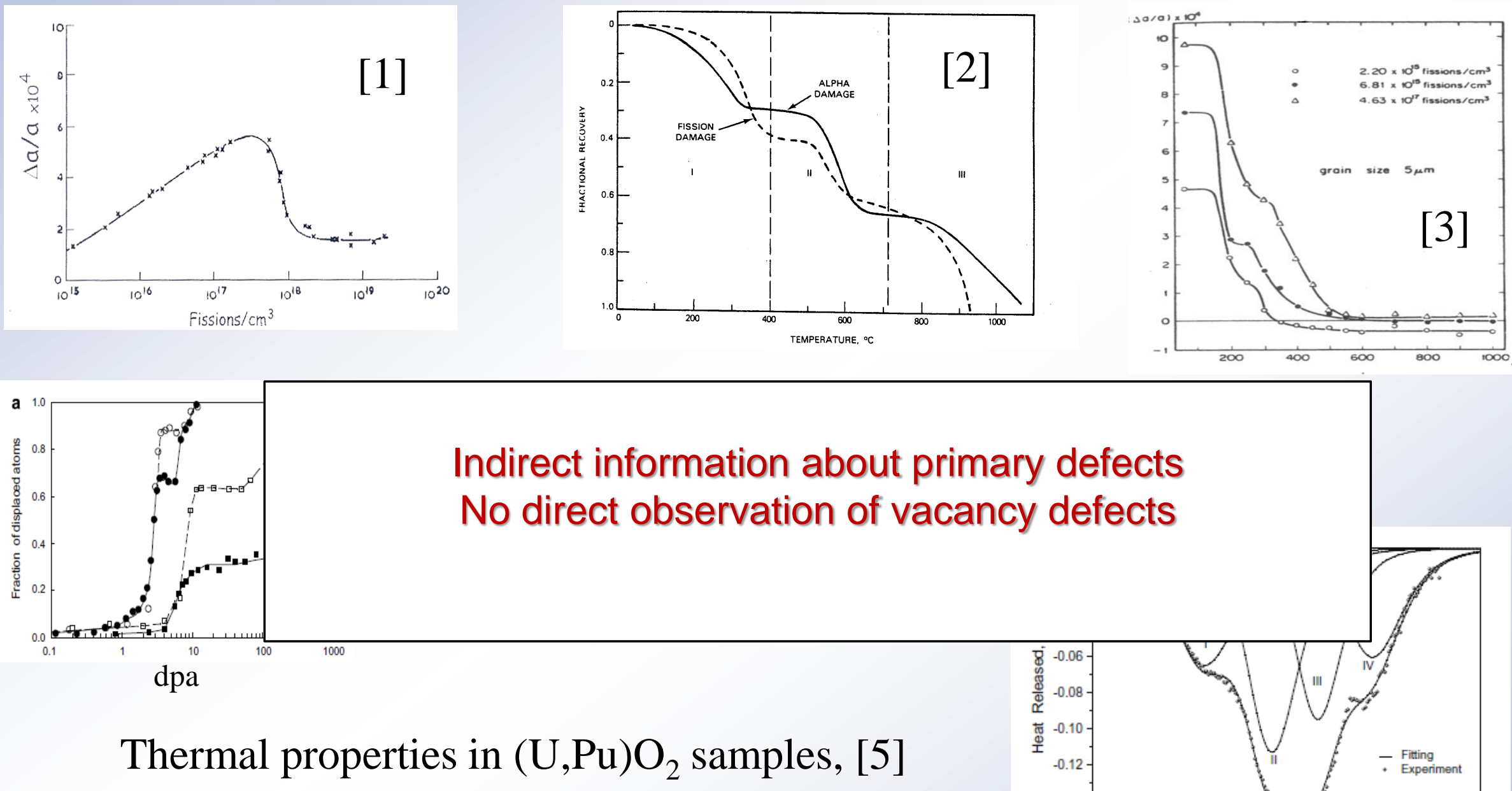

Indirect information about primary defects No direct observation of vacancy defects

[1] J.H.Davies et al, JNM 41 (1971), 143., [2] Weber W.J, JNM 114 (1983), 213 [3] N.Nakae, JNM 80 (1979), 314-322 , [4] F. Garrido et al. NIM B 266 (2008) 2842-2847, [5] D. Staicu, T. Wiss et al. JNM 397 (2010) 8-18 
Vacancy defects in irradiated $\mathrm{UO}_{2}$
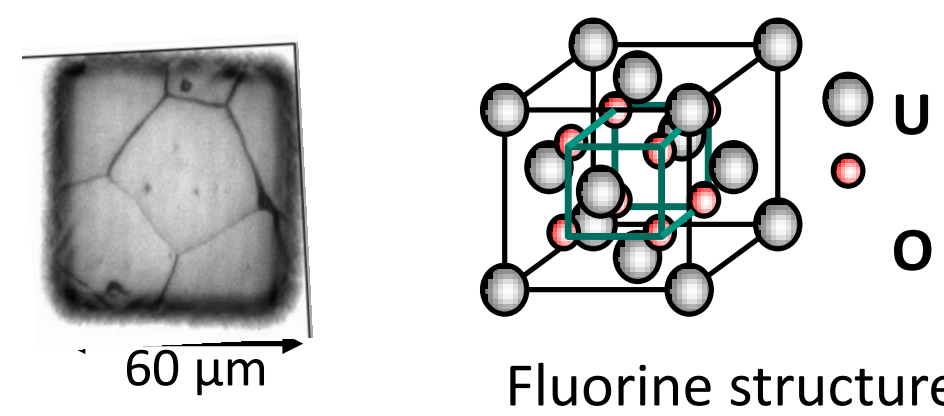

Fluorine structure

$>$ (fast) Positron Lifetime,

$>$ Self-consistent Two Component DFT calculations of positron lifetime
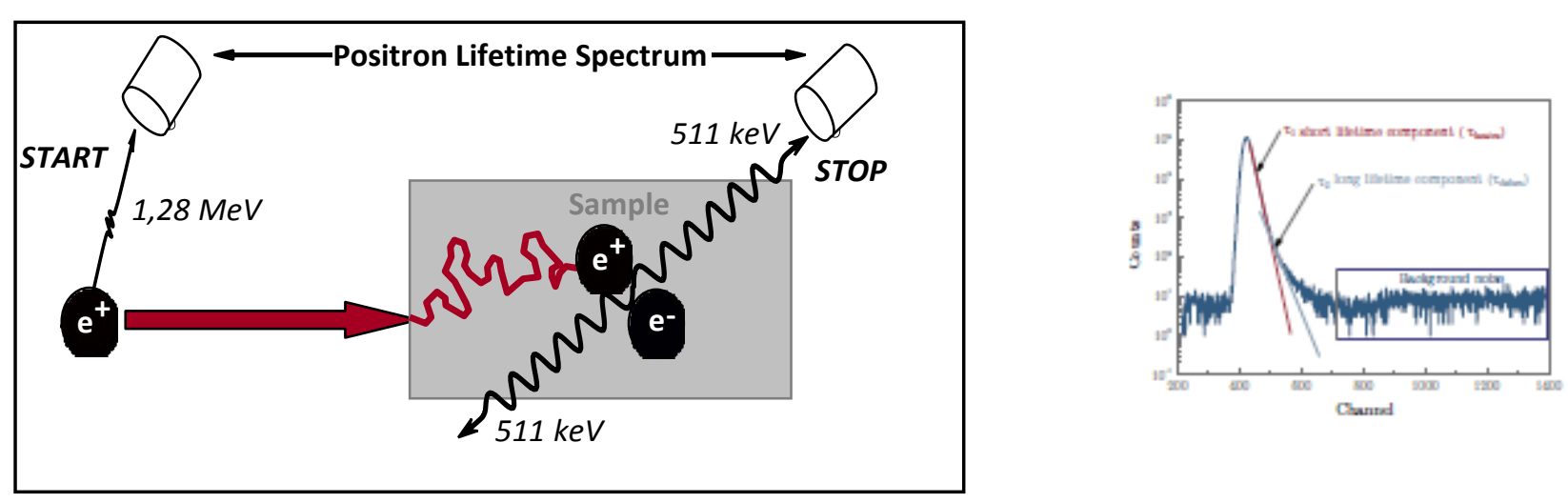


\begin{tabular}{|c|c|c|c|c|c|c|c|}
\hline \multirow[t]{2}{*}{ Sample } & \multirow[t]{2}{*}{ Density (\%) } & \multicolumn{2}{|l|}{ Dopant } & \multicolumn{2}{|c|}{ Integrated dose before thermal treatment } & \multicolumn{2}{|c|}{ Annealing } \\
\hline & & Nature & Conc. (wt.s) & $\left(\alpha g^{-1}\right)$ & (dpa) & $T(K)$ & Time (h) \\
\hline $\mathrm{LO}_{2}$-target & 95 & - & - & 0 & 0 & 1973 & 24 \\
\hline $\mathrm{UO}_{2} \mathrm{G} 1 \_1373$ & 95 & & & $1.5 \times 10^{11}$ & & 1373 & 5 \\
\hline $\mathrm{UO}_{2} \mathrm{G} 1 \_1673$ & 95 & & & $1.5 \times 10^{11}$ & & 1673 & 4 \\
\hline $\mathrm{UO}_{2} \mathrm{G} 1 \_1773$ & 95 & & & $1.5 \times 10^{11}$ & & 1773 & 4 \\
\hline B1d/B3d & 95 & ${ }^{239} \mathrm{Pu}$ & 0.2 & $10^{15}$ & $2 \times 10^{-4}$ & None & \\
\hline B1d_1373/B3d_1373 & 95 & ${ }^{299} \mathrm{Pu}$ & 0.2 & $10^{15}$ & $2 \times 10^{-4}$ & 1373 & 6 \\
\hline GIGONDAS & 96 & ${ }^{209} \mathrm{Pu}$ & 27 & $12 \times 10^{13}$ & 0.3 & None & \\
\hline
\end{tabular}

able 3

ositron lifetime components in $\mathrm{UO}_{2}$ and doped $\mathrm{UO}_{2}$.

\begin{tabular}{|c|c|c|c|c|c|c|c|}
\hline \multirow[t]{2}{*}{ Sample } & \multirow[t]{2}{*}{ Mean lifetime (ps) } & \multicolumn{6}{|c|}{ Lifetime component } \\
\hline & & $\tau_{1}(p s)$ & $I_{1}(\%)$ & $\tau_{2}$ & $I_{2}$ & $\tau_{3}$ & $I_{3}$ \\
\hline $\mathrm{UO}_{2}$ _target & $179.9 \pm 2$ & $179.9 \pm 2$ & 100 & & & & \\
\hline $\mathrm{UO}_{2} \mathrm{G} 1 \_1373$ & $192.8 \pm 2$ & $183 \pm 2$ & 85 & 230 & 14.7 & $2195 \pm 430$ & 0.3 \\
\hline $\mathrm{UO}_{2} \mathrm{G} 1 \_1673$ & $178.1 \pm 1$ & $177.3 \pm 2$ & 98.5 & 230 & 1.5 & & \\
\hline $\mathrm{UO}_{2} \mathrm{G1}{ }_{-1773}$ & $169.2 \pm 0.3^{2}$ & $169.2 \pm 0.3$ & 100 & & & & \\
\hline B1d/B3d & $240.3 \pm 2$ & $204.5 \pm 7$ & 69.5 & $313 \pm 19$ & 30.4 & $3176 \pm 2056$ & 0.1 \\
\hline B1d_1373/B3d_1373 & $219.6 \pm 1$ & $200 \pm 2$ & 93.76 & $446.5 \pm 55$ & 5.8 & 1798 & 0.3 \\
\hline Gigondas & $352.6 \pm 1$ & $222.4 \pm 14$ & 41.3 & $340.6 \pm 14$ & 54.9 & $1916 \pm 43$ & 3.9 \\
\hline
\end{tabular}

[1] D. Roudil, M F Barthe et al. / Journal of Nuclear Materials 420 (2012) 63-68 
Irradiations $\alpha 45 \mathrm{MeV}\left(2 \times 10^{-4} \mathrm{dpa}\right), 1 \mathrm{MeV}$ and $2.5 \mathrm{MeV}$ electrons Positron Annihilation Spectroscopy (Fast Positrons), 300K Lifetime

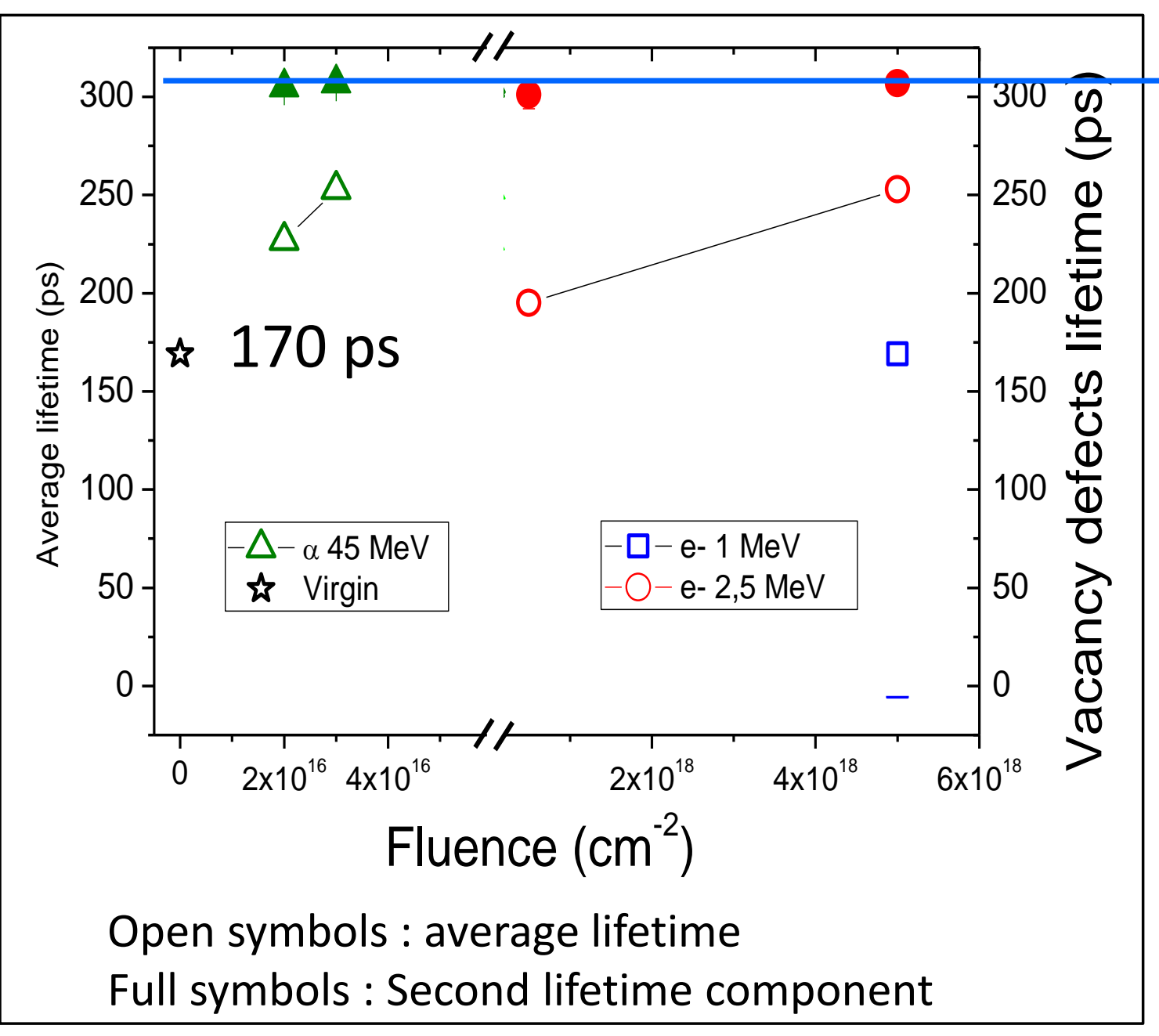

$$
\tau_{\mathrm{v}}=307 \pm 4 \mathrm{ps}
$$

No vacancy defects are detected for $1 \mathrm{MeV}$ e- irradiation

Vacancy defects detected if $2.5 \mathrm{MeV}$ e- irradiation 
Irradiations $\alpha 45 \mathrm{MeV}, 1 \mathrm{MeV}$ and $2.5 \mathrm{MeV}$ electrons

Positron Annihilation Spectroscopy (Fast Positrons), 300K Lifetime

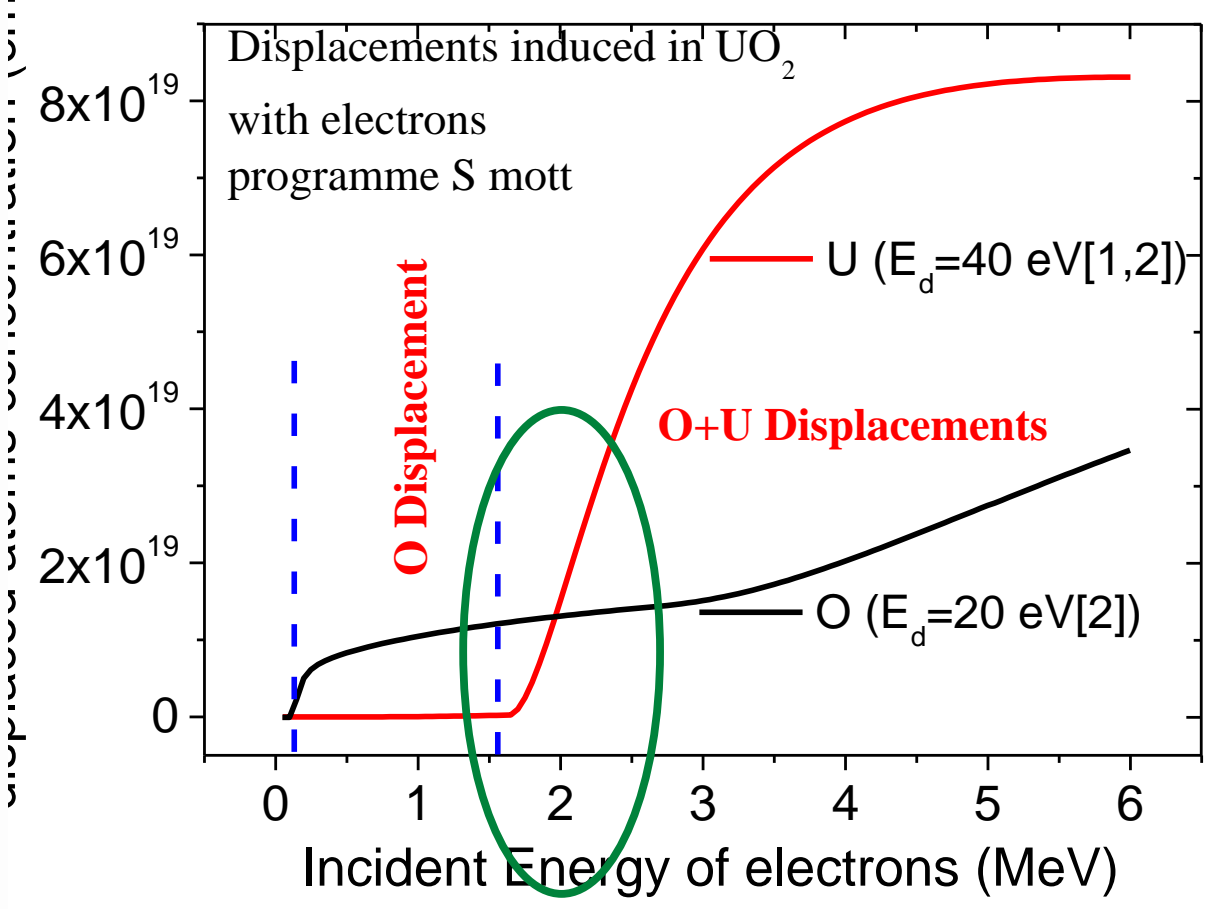

[1] J.Soullard, JNM 135 (1985) 190-196.;[2] C. Meis, A. Chartier JNM 341 (2005) 25

$$
\tau_{\mathrm{v}}=307 \pm 4 \mathrm{ps}
$$

No vacancy defects are detected for $1 \mathrm{MeV}$ e- irradiation

Vacancy defects detected if $2.5 \mathrm{MeV}$ e- irradiation

Displacement of $U$ if $E(e-)>2 M e V$ $\mathrm{V}_{\mathrm{U}}$, or complexes $\mathbf{V}_{\mathrm{U}} \mathbf{2} \mathbf{V}_{\mathrm{O}}$

M F Barthe, et al Phys. Stat. Solidi C 4 3627-3632 2007 


\section{Cemhti Fully self-consistent $\mathbf{2}$ components DFT calculations}

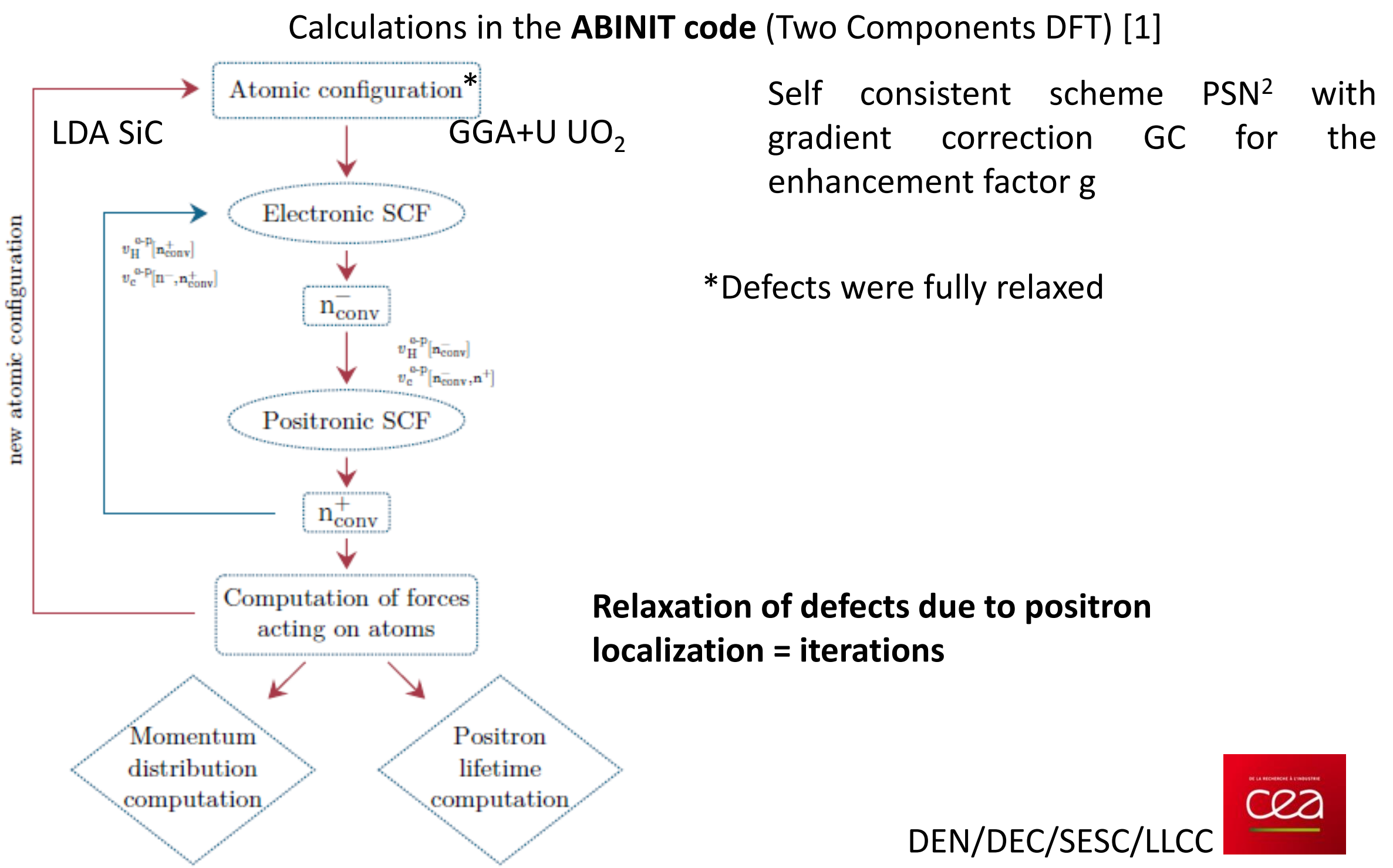

[1] J Wiktor, PhD thesis, University of Marseille, oct 2015, [2] M. J. Puska, A. P. Seitsonen, and R. M. Nieminen, Phys. Rev. B 52, 10947 (1995). 


\section{Identification of vacancy defects in $\mathrm{UO}_{2} \mathrm{irr}$}

\begin{tabular}{llr}
\hline \hline & Charge & $\begin{array}{r}\text { Lifetime } \\
\text { PSN }+\mathrm{GC} \\
(\mathrm{ps})\end{array}$ \\
& & $\begin{array}{r}167 \\
\text { Lattice }\end{array}$ \\
$\mathrm{V}_{\mathrm{O}}$ & 0 & 199 \\
$\mathrm{~V}_{\mathrm{O}}$ & $2-$ & 195 \\
$\mathrm{~V}_{\mathrm{U}}$ & 0 & $\mathbf{3 0 4}$ \\
$\mathrm{V}_{\mathrm{U}}$ & $4-$ & $\mathbf{2 9 3}$ \\
$\mathrm{V}_{\mathrm{U}}+\mathrm{V}_{\mathrm{O}}$ & 0 & $\mathbf{3 0 6}$ \\
$\mathrm{V}_{\mathrm{U}}+\mathrm{V}_{\mathrm{O}}$ & $2-$ & $\mathbf{3 0 1}$ \\
$\mathrm{V}_{\mathrm{U}}+2 \mathrm{~V}_{\mathrm{O}}(100)$ & 0 & $\mathbf{3 0 4}$ \\
$\mathrm{V}_{\mathrm{U}}+2 \mathrm{~V}_{\mathrm{O}}(110)$ & 0 & $\mathbf{3 1 3}$ \\
$\mathrm{V}_{\mathrm{U}}+2 \mathrm{~V}_{\mathrm{O}}(111)$ & 0 & $\mathbf{3 1 6}$ \\
$2 \mathrm{~V}_{\mathrm{U}}$ & 0 & $\mathbf{3 1 8}$ \\
$2 \mathrm{~V}_{\mathrm{U}}$ & $8-$ & $\mathbf{2 8 9}$ \\
$2 \mathrm{~V}_{\mathrm{U}}+2 \mathrm{~V}_{\mathrm{O}}$ & 0 & 339 \\
$2 \mathrm{~V}_{\mathrm{U}}+2 \mathrm{~V}_{\mathrm{O}}$ & $4-$ & $\mathbf{3 1 9}$ \\
$2 \mathrm{~V}_{\mathrm{U}}+4 \mathrm{~V}_{\mathrm{O}}$ & 0 & 329 \\
$2 \mathrm{~V}_{\mathrm{U}}+4 \mathrm{~V}_{\mathrm{O}}$ & $2-$ & $\mathbf{3 6 5}$ \\
\hline \hline & &
\end{tabular}

Exp .

Long lifetime $\sim 310 \pm 10$ ps 
$\mathrm{UO}_{2}$ (set G, sintered polished and annealed $1700^{\circ} \mathrm{C} / 24 \mathrm{~h} / \mathrm{ArH}_{2}$ ) irr alpha $45 \mathrm{MeV} 2 \times 10^{16} \mathrm{~cm}^{-2}$ Measurement in ultra vacuum as a function of temperature

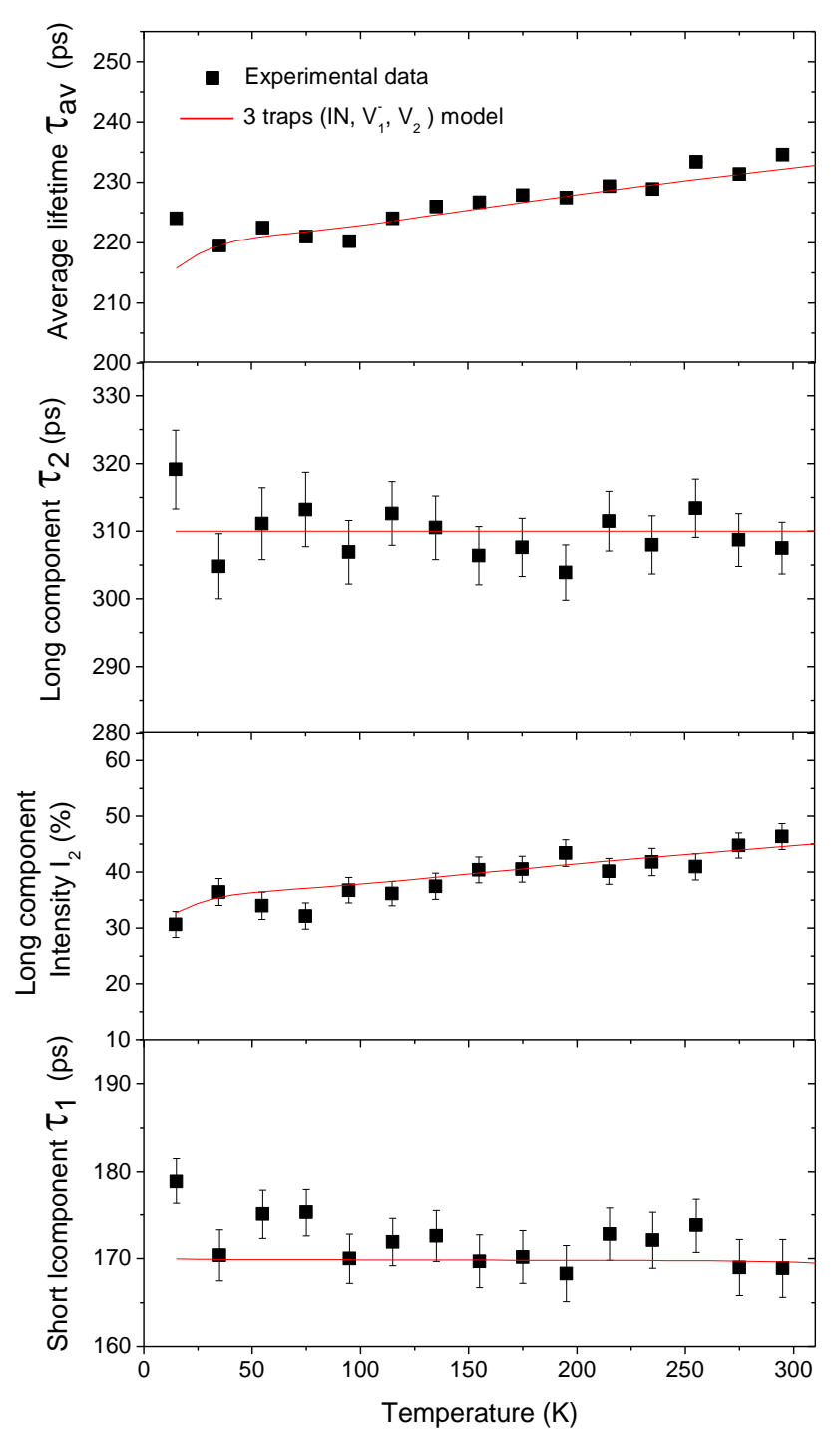

Model with 3 Traps : negative ions IN, $\mathrm{V}_{1}^{-}$, et $\mathrm{V}_{2}$ $\tau_{\mathrm{IN}}=170 \mathrm{ps}$ and $\tau_{\mathrm{V}_{-}}=\tau_{\mathrm{V}_{2}}=310 \mathrm{ps}$
$>30$ times more neutral
\begin{tabular}{l|ll}
$\mathrm{Cv} 2\left(\mathrm{~cm}^{-3}\right)$ & $\mu \mathrm{v} 1$ & vacancies than negatively
\end{tabular}
\begin{tabular}{l|l}
$\mathbf{6}, 50 \mathrm{E}+19$ & $1,00 \mathrm{E}+15$
\end{tabular} charged ones

\begin{tabular}{c|l|l|l|l}
$\mathrm{Cv}-\left(\mathrm{cm}^{-3}\right)$ & $\mu_{0} r$ & $\mu_{0} 2$ & $\mathrm{Er}(\mathrm{eV})$ & $\operatorname{Vr}\left(\mathrm{s}^{-1}\right)$ \\
\hline $\mathbf{2 . 0 E}+18$ & $3,40 \mathrm{E}+16$ & $4,00 \mathrm{E}+16$ & $1,00 \mathrm{E}-02$ & $1,00 \mathrm{E}+11$
\end{tabular}

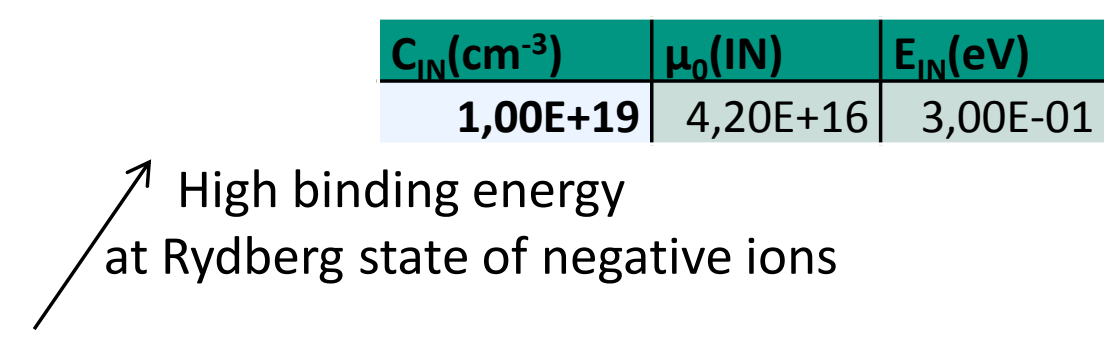

short lifetime is constant

Trapping saturation 
Formation energies of defects in stoichiometric $\mathrm{UO}_{2}[1]$

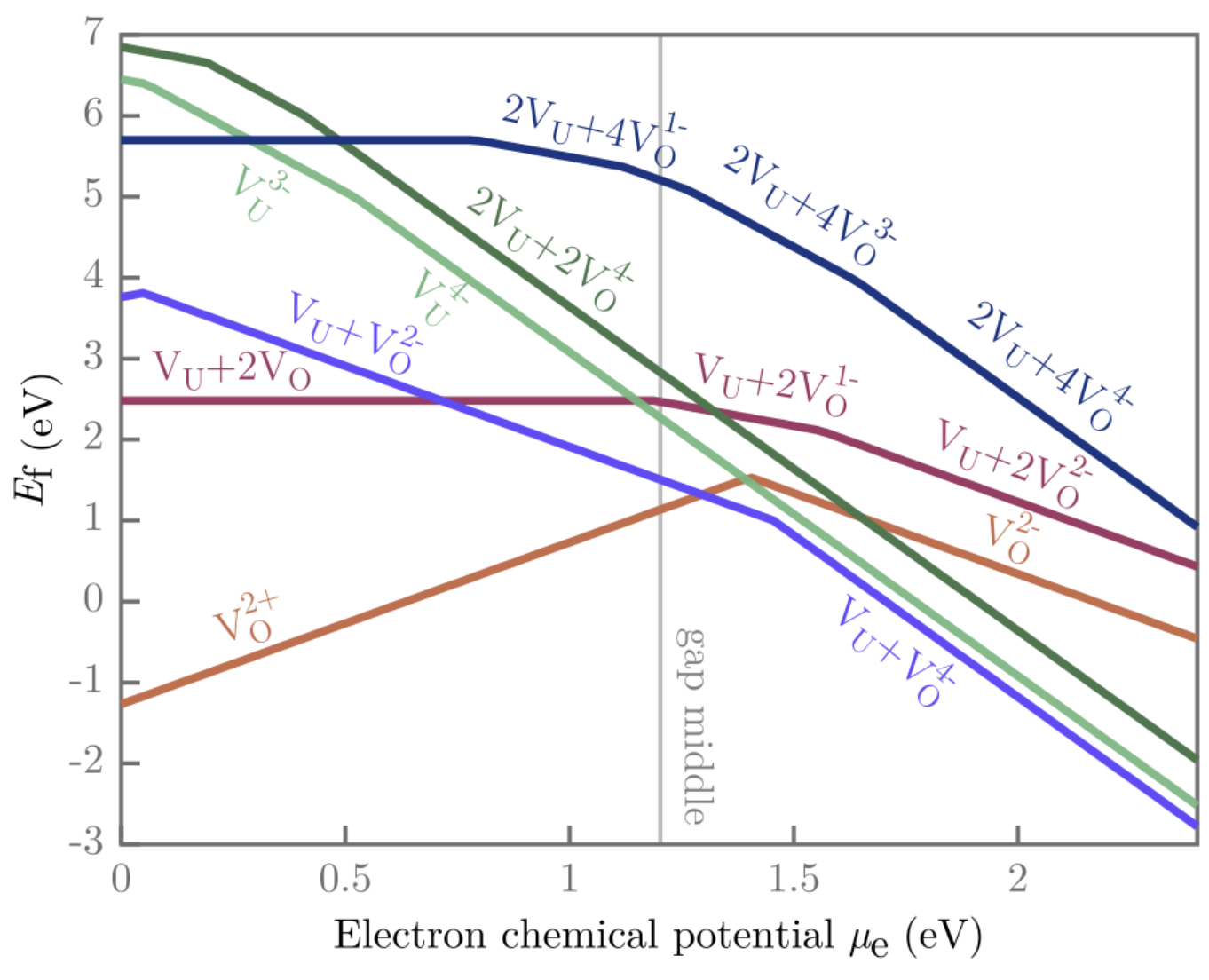

In the undoped $\mathrm{UO}_{2} \mathrm{~V}_{\mathrm{O}}$ should be positive, hence not detected by PAS

$\mathrm{V}_{\mathrm{U}}$ and $\mathrm{V}_{\mathrm{U}}+\mathrm{V}_{\mathrm{O}}$ defects should be negative, while the $\mathrm{V}_{\mathrm{u}}+2 \mathrm{~V}_{\mathrm{O}}$ should be neutral.

DEN/DEC/SESC/LLCC

Close to stoechiometry , $\mathrm{I}_{\mathrm{O}}^{2-}, \mathrm{V}_{\mathrm{O}^{2+}} \mathrm{V}_{\mathrm{U}^{4-}} \mathrm{DFT} L \mathrm{LA}+\mathrm{U}$ [2]

[1] 1 J Wiktor, M. Bertolus, G. Jomard, MF Barthe et al, Phys Rev B 90, 184101 (2014), [2] J P Crocombette Phys Rev B 85, 144101 (2012) 


\begin{tabular}{|c|c|c|}
\hline & Charge & $\begin{array}{r}\text { Lifetime } \\
\mathrm{PSN}+\mathrm{GC} \\
(\mathrm{ps})\end{array}$ \\
\hline Lattice & & 167 \\
\hline $\mathrm{V}_{\mathrm{O}}$ & 0 & 199 \\
\hline $\mathrm{V}_{\mathrm{O}}$ & $2-$ & 195 \\
\hline $\mathrm{V}_{\mathrm{U}}$ & 0 & 204 \\
\hline $\mathrm{V}_{\mathrm{U}}$ & $4-$ & 293 \\
\hline $\mathrm{V}_{\mathrm{U}}+\mathrm{V}_{\mathrm{O}}$ & 0 & (2) \\
\hline $\mathrm{V}_{\mathrm{U}}+\mathrm{V}_{\mathrm{O}}$ & $2-$ & 301 \\
\hline $\mathrm{V}_{\mathrm{U}}+2 \mathrm{~V}_{\mathrm{O}}(100)$ & 0 & $\overline{304}$ \\
\hline $\mathrm{V}_{\mathrm{U}}+2 \mathrm{~V}_{\mathrm{O}}(110)$ & 0 & 313 \\
\hline $\mathrm{V}_{\mathrm{U}}+2 \mathrm{~V}_{\mathrm{O}}(111)$ & 0 & 316 \\
\hline $2 \mathrm{~V}_{\mathrm{U}}$ & 0 & $8+8$ \\
\hline $2 \mathrm{~V}_{\mathrm{U}}$ & $8-$ & 289 \\
\hline $2 \mathrm{~V}_{\mathrm{U}}+2 \mathrm{~V}_{\mathrm{O}}$ & 0 & 339 \\
\hline $2 \mathrm{~V}_{\mathrm{U}}+2 \mathrm{~V}_{\mathrm{O}}$ & $4-$ & 319 \\
\hline $2 \mathrm{~V}_{\mathrm{U}}+4 \mathrm{~V}_{\mathrm{O}}$ & 0 & 329 \\
\hline $2 \mathrm{~V}_{\mathrm{U}}+4 \mathrm{~V}_{\mathrm{O}}$ & $2-$ & 365 \\
\hline
\end{tabular}

\section{$V_{U}$ and $V_{U}-V_{O}$ are negatively charged} close to the middle of the bandgap in close stoechiometric $\mathrm{UO}_{2}$

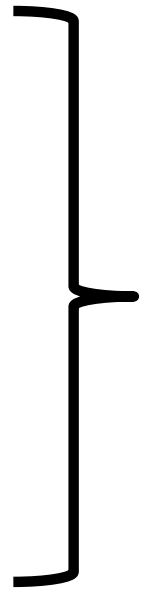

Exp .

Long lifetime $\sim 310 \pm 10$ ps Vacancy defects

$2 V_{u}$ is 8-close to the middle of the bandgap in close stoechiometric $\mathrm{UO}_{2}$

We propose

Neutral vacancy defects which are preponderant $=$ Schottky defects Negatively charged vacancy defects = a mixing of $\left(\mathrm{V}_{\mathrm{U}^{-}}-\mathrm{V}_{\mathrm{O}}\right)^{2-},\left(2 \mathrm{~V}_{\mathrm{U}}-2 \mathrm{~V}_{\mathrm{O}}\right)^{4-}$ 
Positron annihilation spectroscopy

- Vacancy defects properties (nature, migration, agglomeration...) concentration

- From single vacancy to V-clusters

- Thin layers (damage region with HI) / thick samples (electron or high energy LI)

- DFT Calculations of annihilation characteristics

- Complementarity of Lifetime and Doppler

- Complementarity with TEM

In tungsten

- Light ions : single vacancies

$\checkmark$ from $T$ in the range 473-623K: migration and clustering

- Heavy ions

$\checkmark$ at RT or lower temperature

$>$ low dpa : V-clusters (3D small voids + V-loops ) + single vacancies

$>$ high dpa : V-clusters with larger size + single vacancies

$>423 \mathrm{~K}$ clustering due to migration of single vacancies and with $\mathrm{V}$-loops as precurssors?

- He Plasma

$\mathrm{nV}$-mHe complexes close to the surface

In UO2

- Identification of the dominant vacancy defect detected : Schottky defect $\mathrm{V}_{\mathrm{U}}+2 \mathrm{~V}_{\mathrm{O}}$ 


\section{Thank to}

$\checkmark$ All collaborators

$M$ SIDIBE, CEMHTI CNRS

PE LHUILLIER, CEMHTI CNRS

H LABRIM, CEMHTI CNRS

P DESGARDIN, CEMHTI CNRS

C. GENEVOIS-MAZELLIER, CEMHTI CNRS

E. AUTISSIER, CEMHTI CNRS

T SAUVAGE, CEMHTI CNRS

B DECAMPS, CSNSM CNRS

R SCHAUBLIN, ETHZ

A DE BACKER, UMET

C BECQUART, UMET

P TROCELLIER, CEA/SRMP

Y SERRUYS, CEA/SRMPS
J. WIKTOR, CEA/SESC/LLCC

M BERTOLUS, CEA/SESC/LLCC

G CARLOT, CEA/SESC/LLCC

M FREYSS, CEA/SESC/LLCC

P GARCIA, CEA/SESC/LLCC

G JOMARD, CEA/SESC/LLCC

$S$ ESNOUF, LSI

D ROUDIL, CEA/SECM/LMPA

C VALOT, CEA/SESC/LLCC

F VELLA, CEA/SECM/LMPA

$\checkmark$ Accelerators teams Jannus (Orsay and Saclay), LSI, CEMHTI

\section{Thank You for your kind attention}

